M. Pontier

Nagoya Math. J.

Vol. 118 (1990), 1-33

\title{
FILTRAGE APPROCHE ET CALCUL STOCHASTIQUE NON CAUSAL
}

\author{
MONIQUE PONTIER
}

\section{$\S$ I. Introduction}

On considère un signal $X$ partiellement observé par un processus vectoriel $Y$, où la mesure $H$ du signal dépend de l'observation:

$$
Y_{t}=y+\int_{0}^{t} H\left(X_{s}, Y_{s}\right) d s+B_{t}, y \in \boldsymbol{R}^{d}, t \in[0, T] \text {. }
$$

On note $\mathscr{G}^{Y}$ la filtration naturelle associée à $Y$ et $\mathscr{G}^{X}$ celle associée à $X$. On cherche à calculer pour toute variable $Z \mathscr{G}_{T}^{X}$-mesurable et bornée (par exemple $Z=g\left(X_{T}\right)$ ) son espérance conditionnelle sachant $Y: E\left(Z / \mathscr{G}_{T}^{Y}\right)$. Pour cela, on l'approche par une fonction des observations prises à des temps discrets, soit, $h$ étant fixé: $h, 2 h, \cdots, N h$, avec $N$ partie entière de $T / h$, notée $[T / h]$.

On trouve de telles approximations par exemple dans [2] ou [5]. Dans [11], on applique ces procédés d'approximations au cas où l'observation prend ses valeurs dans une variété compacte. On suit ici partiellement la démarche de Picard [10] qui permet d'obtenir une meilleure vitesse que dans les papiers sus-cités, du moins dans le cas où la mesure du signal $H$ introduite en (1.1) n'est fonction que du signal. Néanmoins, notre démonstration utilise une méthode complètement différente du fait que l'approximation introduite fait intervenir une fonction anticipante; alors, ou bien on utilise les théories de grossissement de filtration comme le fait J. Picard, ou bien il faut définir l'intégrale stochastique de processus non adaptés comme le font par exemple E. Pardoux et P. Protter dans [9] ou utiliser l'intégrale de "Skorohod" qui s'appuie sur le calcul dit de Malliavin, ou "non-causal", (voir [4], [7], [8], [13], [14], [15] par exemple). C'est ce dernier choix qui est fait ici.

On suppose que le "bruit" $B$ figurant dans l'observation (1.1) est

Received January 5, 1988. 
indépendant du signal $X$. On construira le modèle dans le paragraphe suivant sur un espace de probabilité $(\Omega, \mathscr{A}, \boldsymbol{Q})$ muni d'une filtration $\mathscr{G}$ dont $\mathscr{G}^{X}$, filtration naturelle associée à $X$, et $\mathscr{G}^{Y}$, filtration naturelle associée à $Y$, sont des sous-filtrations. Puis on montrera l'existence d'une probabilité $\boldsymbol{P}$, équivalente à $\boldsymbol{Q}$, sous laquelle $X$ et $Y$ sont indépendants. L'approximation proposée pour le filtre d'une variable aléatoire $Z \mathscr{G}_{T}^{X}$ mesurable, noté $\pi_{T}(Z)$ et défini par $E_{Q}\left(Z / \mathscr{G}_{T}^{Y}\right)$, est de la forme:

$$
\pi^{h}(Z)=E_{P}\left(L_{N}^{h} Z / \mathscr{G}_{T}^{Y}\right) / E_{\boldsymbol{P}}\left(L_{N}^{h} / \mathscr{G}_{T}^{Y}\right)
$$

où la variable $L_{N}^{h}$ est définie par:

$$
L_{N}^{h}=\exp \sum_{i=1, N}\left(H_{i h}\left(Y_{i h}-Y_{(i-1) h}\right)-h / 2\left\|H_{i h}\right\|^{2}-h \operatorname{div} H_{i h}\right) ;
$$

$H_{i h}$ note $H\left(X_{i h}, Y_{i h}\right)$ et $\partial_{j} H=\partial H(x, y) / \partial y_{j}$.

Ainsi, dans le paragraphe suivant, on construit le modèle, on définit la probabilité $\boldsymbol{P}$ et on démontre l'indépendance de $X$ et $Y$ sous $\boldsymbol{P}$; de plus, on y précise les hypothèses. Le troisième paragraphe contient quelques rappels du calcul stochastique "non causal" [7] et la démonstration de la convergence de $\pi^{h}(Z)$ vers $\pi_{T}(Z)$ est donnée dans le dernier paragraphe avec une majoration de l'erreur dans les deux cas suivants: $H$ est une fonction bornée, ou bien: $H$ ne dépend que du signal.

\section{§ II. Hypothèses et construction du modèle}

L'hypothèse d'indépendance entre le "bruit" $B$ et le signal $X$ permet de construire le modèle du système signal-observation de la façon suivante: on considère séparément l'espace de probabilité canonique associé à $B$, muni de la mesure de Wiener $\mu$ :

$$
(W, B(W), \mu)
$$

où $W$ est l'ensemble des fonctions continues sur $[0, T]$ nulles en 0 à valeurs dans $\boldsymbol{R}^{d}$, muni de la tribu de ses boréliens: $B(W)$, et l'espace de probabilité du signal $X$ :

$$
\left(\Omega^{x}, \mathscr{A}, \boldsymbol{P}^{X}\right)
$$

où $\Omega$ est l'ensemble des fonctions continues sur $[0, T]$ nulles en 0 à valeurs dans $\boldsymbol{R}^{n}$ dont $\mathscr{A}$ est la tribu des boréliens. On supposera que $X$ est une diffusion à valeurs dans une variété $E$, dirigée par les champs de vecteurs $\left(A_{0}, A_{\alpha} ; \alpha=1, \cdots, n\right)$ (cf. [3] p. 234) et conduite par un brownien $V$ dont 
$\left(\Omega^{x}, \mathscr{A}, \boldsymbol{P}^{x}\right)$ est l'espace de probabilité canonique:

$$
d X_{t}=A_{a}(X) o d B_{t}^{\alpha}+A_{0}\left(X_{t}\right) d t .
$$

On note $\mathscr{G}^{X}=\sigma\left\{X_{s} ; s \leq t\right\}$ et l'on considère l'espace de probabilité produit:

$$
\Omega=\Omega^{x} \times W ; \mathscr{G}=\mathscr{A} \otimes B(W) ; \boldsymbol{Q}=\boldsymbol{P}^{x} \otimes \mu,
$$

c'est à dire que l'on prolonge $B$ et $V$ sur $\Omega: B(v, w)=w(t)$ et $V(v, w)=$ $v(t)$.

On identifie alors la filtration $\mathscr{G}^{X}$ à $\mathscr{G}^{X} \otimes \mathscr{B}(W)$, sous-filtration de la filtration $\mathscr{G}$ associée au couple $(X, B)$ sur $\Omega: \mathscr{G}=\sigma\left\{\left(X_{s}, B_{s}\right) ; s \leq t\right)$, on note de même $\mathscr{G}^{Y}$ la filtration naturelle associée à $Y$, solution de l'équation différentielle stochastique (1.1) définie sur $\Omega$. De façon classique, sans changer les notations, on complète par rapport à $\boldsymbol{Q}$ et on rend continues à droite les filtrations $\mathscr{G}^{X}$ et $\mathscr{G}^{Y}$.

On suppose que la fonction $H$ du signal vérifie:

(2.4) $H \in C^{2}\left(E \times \boldsymbol{R}^{d}\right)$ et ses dérivées notées $A_{\alpha} H, A_{\alpha}\left(A_{\alpha} H\right), \partial_{i} H, \partial_{i j} H$ sont uniformément bornées.

On notera dans toute la suite $I_{i}$ l'intervalle ] $\left.(i-1) h, i h\right], i$ allant de 1 à $N$, et $H^{h}$ le processus constant et égal à $H_{i n}$ sur $I_{2}$. On fera l'étude dans les deux cas suivants:

A. $H$ est uniformément borné;

B. $H$ est uniquement fonction de $X$

On utilisera dans toute la suite les notations suivantes: par abus de langage, on notera $\Omega$ aussi bien le brownien $(B, V)$ que l'espace de probabilité; de plus, $H$ désignera également le processus $t \rightarrow H\left(X_{t}, Y_{t}\right)$; enfin, l'hypothèse (2.4) assure l'existence de processus $b$ et $\sigma$ tels que:

$$
H_{t}=H_{0}+\int_{0}^{t} b_{s} d s+\int_{0}^{t} \sigma_{s} d \Omega_{s}, \quad t \in[0, T],
$$

où

$$
\begin{aligned}
b_{t}= & \left(A_{0} H\right)\left(X_{t}, Y_{t}\right)+1 / 2\left(A_{\alpha}\left(A_{\alpha} H\right)\right)\left(X_{t}, Y_{t}\right)+\left(\partial_{y} H \cdot H\right)\left(X_{t}, Y_{t}\right) \\
& +1 / 2 \partial_{i i} H\left(X_{t}, Y_{t}\right) \\
\sigma_{t}= & {\left[\begin{array}{c}
\left(A_{\alpha} H\right)\left(X_{t}, Y_{t}\right) \\
\partial_{y} H\left(X_{t}, Y_{t}\right)
\end{array}\right] }
\end{aligned}
$$

Il est clair que dans le cas B n'intervient dans l'intégrale stochastique que la composante $B$ de $\Omega$; de plus, dans les deux cas, $b$ et $\sigma$ sont bornés; on suppose enfin que $H_{0}$ appartient à $L^{q}(\Omega, \boldsymbol{Q})$ pour tout $q$. 
Remarque. Ces hypothèses ne sont guère différentes dans le cas $B$ de celles de Picard [10], qui suppose l'existence de $b$ et $\sigma$ et de tous les moments de $H_{0}, \int_{0}^{T}\left\|b_{s}\right\|^{2} d s$ et de $\sup _{t}\left\|\sigma_{t}\right\|$.

Ceci étant, que l'on soit sous l'une ou l'autre des hypothèses $\mathrm{A}$ ou B, (2.4)-(2.5) montrent qu'il est possible de définir une $(\mathscr{G}, \boldsymbol{Q})$-martingale uniformément intégrable et strictement positive:

$$
L_{t}=\exp \int_{0}^{t}\left(-H_{s} \cdot d B_{s}-1 / 2\left\|H_{s}\right\|^{2} d s\right)
$$

en sorte que la probabilité $\boldsymbol{P}=L_{T} \cdot \boldsymbol{Q}$ est équivalente à $\boldsymbol{Q}$. De plus, le théorème de Girsanov montre que $Y$ (1.1) suit la loi d'un mouvement brownien sous la probabilite $\boldsymbol{P}$. On montre alors:

Lemme 2.1. Sous les hypothèses précédentes, les processus $X$ et $Y$ sont indépendants sur l'espace de probabilité $(\Omega, \mathscr{G}, \boldsymbol{P})$.

Démonstration. Il suffit de démontrer que pour toute fonction $g$ bornée sur $[0, T]$, et pour tout $k$, tout $k$-uple $\left(t_{1}, \cdots, t_{k}\right)$ et toute fonction $f$ bornée sur $E^{k}$, l'égalité suivante est vérifiée:

$$
\begin{aligned}
E_{P}\left(f \left(X_{t_{1}}, \cdots\right.\right. & \left.\left., X_{t_{k}}\right) \exp i \int_{0}^{T} g_{s} d Y_{s}\right) \\
& =E_{P}\left(f\left(X_{t_{1}}, \cdots, X_{t_{k}}\right)\right) E_{P}\left(\exp i \int_{0}^{T} g_{s} d Y_{s}\right)
\end{aligned}
$$

On note $\varphi_{X, Y}(f, g)$ le membre de gauche et on le calcule en utilisant la définition (2.6).

$$
\begin{aligned}
& \varphi_{X, Y}(f, g) \\
& \quad=E_{Q}\left(f\left(X_{t_{i}}\right) \exp \int_{0}^{T}\left(-H_{s} d B_{s}-1 / 2\left\|H_{s}\right\|^{2} d s+i g_{s} d B_{s}+i g_{s} H_{s} d s\right)\right)
\end{aligned}
$$

L'indépendance de $X$ et $\mathrm{B}$ sous $\boldsymbol{Q}$ permet de conditionner par $\mathscr{G}_{T}^{X}$ sous l'espérance et de sortir $f\left(X_{t_{i}}\right)$ :

$$
\begin{aligned}
\varphi_{X, Y}(f, g)=E_{Q}\left(f ( X _ { t _ { i } } ) E _ { Q } \left[\exp \int_{0}^{T}\left(-H_{s}+i g_{s}\right) d B_{s}\right.\right. \\
\left.\left.-1 / 2\left\|-H_{s}+i g_{s}\right\|^{2} d s-1 / 2\left\|g_{s}\right\|^{2} d s / \mathscr{G}_{T}^{X}\right]\right)
\end{aligned}
$$

La variable conditionnelle en $X$ ci-dessus, au facteur déterministe $\exp -1 / 2 \int_{0}^{T}\left\|g_{s}\right\|^{2} d s$ près, est une martingale de mêmes propriétés que $L$ sous les hypothèses choisies; il vient donc: 


$$
\begin{aligned}
E_{Q}\left[\operatorname { e x p } \int _ { 0 } ^ { T } \left(-H_{s}\right.\right. & \left.\left.+i g_{s}\right) d B_{s}-1 / 2\left\|-H_{s}-i g_{s}\right\|^{2} d s-1 / 2\left\|g_{s}\right\|^{2} d s \mid \mathscr{G}_{T}^{X}\right] \\
& =\exp -1 / 2 \int_{0}^{T}\left\|g_{s}\right\|^{2} d s .
\end{aligned}
$$

Du fait que $Y$ suit la loi d'un mouvement brownien sous $\boldsymbol{P}$, le membre à droite de (2.10) est justement $E_{P}\left(\exp i \int_{0}^{T} g_{s} d Y_{s}\right)$.

Cetter remarque et les calculs précédents montrent alors:

$$
\varphi_{X, Y}(f, g)=E_{Q}\left(f\left(X_{t_{1}}, \cdots, X_{t_{k}}\right)\right) E_{P}\left(\exp i \int_{0}^{T} g_{s} d Y_{s}\right) .
$$

Or, si l'on choisit pour $g$ la gonction nulle, la suite des calculs (2.8)(2.11) donne simplement

$$
E_{P}\left(f\left(X_{t_{1}}, \cdots, X_{t_{h}}\right)\right)=E_{Q}\left(f\left(X_{t_{1}}, \cdots, X_{t_{k}}\right)\right)
$$

ce qui, rapproché de (2.11), achève la démonstration de (2.7).

L'équivalence des probabilités $\boldsymbol{P}$ et $\boldsymbol{Q}$ permet de traduire le filtre sous $\boldsymbol{P}$ ou sous $\boldsymbol{Q}$ :

$$
\pi_{T}(Z)=E_{Q}\left(Z / \mathscr{G}_{T}^{Y}\right)=E_{P}\left(L_{T}^{-1} Z / \mathscr{G}_{T}^{Y}\right) / E_{P}\left(L_{T}^{-1} \mid \mathscr{G}_{T}^{Y}\right)
$$

La variable $L_{N}^{h}$ introduite en (1.3) a vocation à approcher la variable $L_{T}^{-1}$. Reppelons ici l'approximation proposée:

$$
\pi^{h}(Z)=E_{P}\left(L_{N}^{h} Z / \mathscr{G}_{T}^{Y}\right) / E_{P}\left(L_{N}^{h} / \mathscr{G}_{T}^{Y}\right)
$$

Remarquons que, grâce à l'indépendance sous $\boldsymbol{P}$ de $X$ et $Y, \pi^{h}(Z)$ est le quotient de fonctions explicites des observations $y_{i}$ :

$$
E_{P}\left(L_{N}^{h} Z / \mathscr{G}_{T}^{Y}\right)=E_{P^{X}}\left[Z\left(\omega^{X}\right) L_{N}^{h}\left(\omega, y_{0}, \cdots, y_{N}\right)\right]
$$

Remarque. On ne peut considérer que cette approximation est un filtre discret comme c'est habituellement le cas ([2], [5]); en effet, du fait de l'anticipation qui figure dans $L_{N}^{h}$ (1.3) (le coefficient de l'accroissement de l'observation dépend de l'instant final), on a besoin, pour obtenir la convergence, du terme correctif $h$. div $H_{i n}$ qui empêche $L_{N}^{h}$ d'être un rapport de vraisemblance entre $\boldsymbol{P}$ et la probabilité qui modéliserait une observation discrète avec fonction anticipante du signal, à savoir: $Y_{i}=$ $Y_{i-1}+h H_{i h}+\Delta_{i} B$.

Dans le paragraphe IV, on montre que $\pi^{h}(Z)$ converge vers $\pi(Z)$ et on estime la vitesse de convergence. Pour ce faire, on utilise le calcul 
des variations stochastiques afin d'exprimer $L_{N}^{h}$ à l'aide de l'intégrale de Skorohod. On utilise dans la suite de ce papier les notations soit de D. Nualart [7], soit de S. Watanabe [14]: le mouvement brownien $B$ donne lieu avec son espace canonique à un espace de Wiener $(W, \mu)$ sur lequel on note l'intégrale de Skorohod ([8] §3) et son opérateur dual dit gradient stochastique [13] ou dérivée de Malliavin ([8] 2.4): $\delta$ et D. On rappelle que $H^{h}$ est le processus constant et égal à $H_{i n}$ sur l'intervalle $I_{i}$ et définit le processus:

$$
L_{t}^{h}=\exp \int_{0}^{T}\left(H_{s}^{h} \delta B_{s}+\left(H_{s}^{h} H_{s}-1 / 2\left\|H_{s}^{h}\right\|^{2}+D_{s} H_{s}^{h}-\operatorname{div} H_{s}^{h}\right) d s\right)
$$

On montrera auparavant que $L_{N}^{h}$ (1.3) coïncide au temps $N h$ avec ce processus $L^{h}$, après avoir donné quelques rappels sur le calcul stochastique "non-causal".

\section{§III. Calcul stochastique non causal}

On considère un espace de Wiener standard $(W, B(W), \mu)$ où $W$ est l'espace des trajectoires vectorielles continues sur $[0, T]$, nulles à l'origine, $B(W)$ la tribu des boréliens et une mesure de Wiener centrée. Dans cette partie, on définit les opérateurs $\delta$ et $D$, intégrale de Skorohod et gradient stochastique déja cités, sur des espaces simples. Puis on donne les définitions des espaces de Sobolev où sont définis ces opérateurs. Ensuite, on rappelle les propriétés de $D$ et $\delta$ qui seront utiles par la suite: régularité, formules d'intégration par parties et de commutation, règles de dérivation, formule de Clark et "formule de Itô" étendue aux processus anticipatifs. On conclut cette partie par la justication du processus $L^{h}$, introduit en (2.15), qui utilise certaines des propriétés rappellées.

1. Introduisons comme le fait Nualart ([7] 3.1) les fonctionelles régulières de Wiener

$$
S=\left\{F=f\left(W\left(t_{1}\right) \cdots W\left(t_{n}\right)\right) ; f \in C_{b}^{\infty}\left(\boldsymbol{R}^{n}\right), t_{i} \in[0, T]\right\}
$$

où $C_{b}^{\infty}$ désigne les fonctions de classe $C^{\infty}$, bornées ainsi que leurs dérivées; $S$ est contenu dans $L^{2}(W)$. On définit pour tout Hilbert séparable $E$, la famille de fonctionnelles:

$$
S^{E}=\left\{u=\sum F_{i} l_{i} ; F_{1} \cdots F_{n} \in S ; l_{1} \cdots l_{n} \in E\right\}
$$

On utilisera surtout $S^{L}$, où $L$ est le Hilbert $L^{2}[0, T]$. 
Il y a plusieurs définitions de l'opérateur $D$, gradient stochastique, qui toutes coïncident de façon évidente sur l'espace $S$, mais dont les espaces de définitions semblent a priori différents. On choisit ici de le définir comme "dérivée directionnelle", ou de Fréchet, le long de $h$, élément de $L$ :

$$
D F(h)=\lim _{\varepsilon \rightarrow 0}\left(F\left(\omega+\varepsilon \int_{0} h_{s} d s\right)-F(\omega)\right) / \varepsilon .
$$

Dans $S$, la limite peut être prise dans tous les sens "classiques" et pour $F$ décrite en (3.1) on tire:

$$
D F(h)=\sum_{i=1, n} \partial_{i} f\left(W\left(t_{1}\right) \cdots W\left(t_{n}\right)\right) \int_{0}^{t_{i}} h_{s} d s
$$

Cette expression permet de mettre en évidence l'élément suivant de $S^{L}$ :

$$
D_{t} F=\sum_{i=1, n} \partial_{i} f\left(W\left(t_{1}\right) \cdots W\left(t_{n}\right)\right) 1_{\left[0, t_{i}\right]}(t)
$$

qui vérifie

$$
D F(h)=\langle D F, h\rangle_{L}
$$

On adopte ici pour $D$ la définition de Kusuoka citée par Sugita [12]: une fonctionnelle de Wiener $F$ est stochastiquement différentiable si la limite (3.3) existe en probabilité sur $(W, B(W), \mu)$ et définit une fonctionnelle mesurable $D F$ à valeur dans $L$ telle que la limite, notée $D F(h)$, vérifie (3.6).

Si l'on peut itérer cet opérateur et obtenir successivement $D^{2} F(h ; k)$ $\cdots D^{k} F\left(h_{i_{1}} \cdots h_{i_{k}}\right)$, on définit:

$$
\left\|D^{k} F(\omega)\right\|_{\text {H.s. }}^{2}=\sum_{i_{1} \cdots i_{k}}\left|D^{k} F(\omega)\left(h_{i_{1}} \cdots h_{i_{k}}\right)\right|^{2}
$$

où $\left\{h_{i}\right\}$ est une base orthonormée de $L$, et pour $F k$ fois stochastiquement différentiable, on définit la semi-norme:

$$
\|F\|_{p, k}=\|\| F\left\|_{E}\right\|_{p}+\|\| D^{k} F\left\|_{\text {H.s. }}\right\|_{p} ; p>1 ; k \in N
$$

où $\|\cdot\|_{p}$ est la norme de $L^{p}(W, \mu)$. Grâce aux "inégalités de Meyer" ([14] théorème 1.8$)$, cette semi-norme est équivalente à la norme $\|\cdot\|_{p, k}$, utilisée par exemple dans [13] ou [14], qui fait intervenir l'opérateur $\mathfrak{Z}$, générateur infinitésimal du processus de Ornstein-Uhlenbeck.

Ceci étant, on considère les fonctionnelles $F$ à valeurs dans un Hilbert séparable $E$, "R.A.C.", c'est-à-dire que pour tout $h$ de $L$, il existe 
une version mesurable de $F\left(\omega+t \int_{0} h_{s} d s\right)$ absolument continue en $t$ (cf. [12]). Soit $\underline{D}(E)$ l'ensemble de ces fonctionnelles qui sont de plus stochastiquement différentiables. On pose alors pour $p>1$ et $k$ entier naturel:

$$
D_{p, k}(E)=\left\{F \in \underline{D}(E) /\|F\|_{E} \text { et }\left\|D^{i} F\right\|_{\text {1r.s. }} \in L^{p}, i=1 \cdots k\right\}
$$

On notera ces espaces $D_{p, k}$ quand $F$ est à valeurs réelles ou vectorielles. Sugita montre [12] que cette définition d'espaces de Sobolev par Kusuoka et Stroock coïncide avec les définitions "habituelles", telles celles données dans [7], [8], [14], ou [15], c'est-à-dire:

$$
D_{p, k}(E) \text { est la complétion de } S^{E} \text { pour la norme }\|\|_{p, k}
$$

et le gradient stochastique $D$ défini par la limite en probabilité (3.3) coïncide avec celui des références ci-dessus, c'est-à-dire que $D F$ est obtenu comme limite de $D F_{n}$, où $F_{n}$ est une suite d'éléments de $S^{E}$ de Cauchy pour la norme $\|\cdot\|_{p, k}$ et convergeant vers $F$. En particulier, \|\|$D F_{n}-D F_{m}\left\|_{\text {H.s. }}\right\|_{p}$ tend vers 0 pour $n$ et $m$ infinis, et pour $E=\boldsymbol{R}$, la norme de Hilbert Schmidt s'écrit simplement en fonction des processus $D_{t} F(3.5)$ :

$$
\left\|D F_{n}-D F_{m}\right\|_{\text {I. } . \mathrm{S} .}^{2}=\int_{0}^{T}\left|D_{t} F_{n}-D_{t} F_{m}\right|^{2} d t .
$$

En passant à la limite, on a donc l'existence d'un processus de $L, D_{t} F$, dont la norme de Hilbert Schmidt appartient à $L^{p}$ et qui vérifie la relation (3.6) pour tout $h$ de $\boldsymbol{L}$.

Par ailleurs, on définit l'opérateur $\delta$, dit intégrale de Skorohod, sur l'espace $S^{L}$, par linéarité à partir de l'image des processus de la forme $F \cdot g, F$ élément de $S$ et $g$ de $L$ :

$$
\delta(F \cdot g)=F \int_{0}^{T} g_{t} d W_{t}-D F(g)
$$

On notera par la suite $\delta(u)$ par: $\int_{0}^{T} u_{t} \delta W_{t}$. Puis, utilisant la norme (3.8) pour le cas $E=L$ et la définition (3.9) de $D_{p, k}(L)$, on définit $\delta$ sur les espaces de Sobolev $D_{p, k}(L)$ par passage à la limite.

Pour montrer qu'un processus est élément de $D_{p, k}$, on se servira le plus souvent de la caractérisation de Kusuoka-Stroock (3.9) qui offre l'avantage d'une vérification plus simple d'appartenance aux espaces de Sobolev. Ceci étant, on utilisera selon le besoin l'une ou l'autre des deux 
définitions (3.9) ou (3.10). Notons simplement ici que pour un processus $u$ de $L$ son gradient stochastique $D u$ est un processus sur $[0, T]^{2}$ de norme de Hilbert-Schmidt:

$$
\|D u\|_{\mathrm{H} . \mathrm{S} .}^{2}=\int_{0}^{T} \int_{0}^{T}\left|D_{s} u_{t}\right|^{2} d s d t
$$

2. On rappelle maintenant quelques propriétés utiles sur les opérateurs $D$ et $\delta$ et les espaces $D_{p, k}$ et $D_{p, k}(L)$. En premier lieu, $D$ et $\delta$ ont une certaine régularité (voir [14] Proposition 1.9):

$D$ est un opérateur continu de $D_{p, k+1}$ dans $D_{p, k}(L)$

$\delta$ est un opérateur continu de $D_{p, k+1}(L)$ dans $D_{p, k}$

pour $p>1$ et $k$ entier naturel. Ils sont de plus en dualité, grâce à la "formule d'intégration par parties" (voir [8] ou [4]):

$$
\text { Si } F \in D_{q, 1} \text { et } u \in D_{p, 1}(L) \text { et } 1 / p+1 / q \leq 1: E(F \delta(u))=E\langle D F, u\rangle_{L}
$$

On peut "dériver" stochastiquement l'intégrale de Skorohod; ceci donne lieu à une "règle de commutation" ([7], [8], [13] ...), si $u$ est un processus tel que $D \delta u$ et $\delta D u$ existent:

$$
D_{t} \delta u-\delta D_{t} u=u_{t}, \quad \forall t \in[0, T] ;
$$

ce qui est par exemple le cas pour $u$ dans $D_{2,2}(L)$.

Les règles de dérivation s'appliquent aux fonctions de fonctionnelles à dérivées bornées ([7], [8], [14]): si $f$ est dérivable sur $\boldsymbol{R}^{d}$ à dérivées bornées, et si $F_{1}, \cdots, F_{d}$ appartiennent à $D_{p, 1}$, alors:

$$
D\left[f\left(F_{1}, \cdots, F_{d}\right)\right]=\sum \partial_{i} f\left(F_{1}, \cdots, F_{d}\right) \cdot D F_{i}
$$

Ce n'est pas le cas du produit. On peut néanmoins montrer sans difficulté (cf. (27) dans [15] et Proposition 1.10 [14]).

Lemme 3.1. Soit $F$ dans $D_{p, k}$ et $G$ dans $D_{q, k}$; alors le produit $F G$

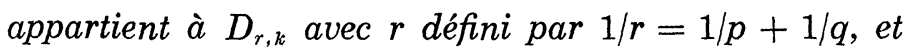

$$
D(F \cdot G)=F \cdot D G+G \cdot D F
$$

De façon plus générale, la définition (3.3) permet d'obtenir la règle de dérivation suivante:

Lemme 3.2. Soit $F$ appartenent $\grave{a} D_{q, 1}, q>1$ et $f$ une fonction de classe $C^{1}$ telle que $f(F)$ et $\nabla f(F)$ appartiennent à $L^{p}(1 / p+1 / q=1 / r<1)$. Alors 
$f(F)$ appartient $\grave{a} D_{r, 1}$ et admet le gradient stochastique:

$$
D(f(F))=\nabla f(F) \cdot D F
$$

La relation (3.18) et la formule d'intégration par parties (3.15) permettent à Nualart ([7] 4.3) de montrer, pour un processus $u$ de $D_{p, 1}(L)$ et $F$ élément de $D_{q, 1}(1 / p+1 / q<1)$ :

$$
\delta(F u)=F \delta(u)-\int_{0}^{T} u_{t}\left(D_{t} F\right) d t
$$

qui prolonge la définition (3.12) de $\delta$; la démonstration consiste simplement à intégrer le produit de chacun des membres de (3.20) avec une fonctionnelle $G$ de $S$ et à montrer l'égalité des deux espérances pour tout $G$.

3. On a considéré jusqu' à présent $W=C([0, T], \boldsymbol{R})$; on peut duppliquer cet espace $n$ fois et obtenir des trajectoires dans $\boldsymbol{R}^{n}$. On notera $\delta^{i}, D^{i}$ les opérateurs correspondant à la $i^{\circ}$ composante de $W$. Ces notations sont utilisées dans le résultat suivant qui est une version de la formule de Clark pour les fonctionnelles de $D_{2,1}$ donnée par Nualart dans ([7] 3.12); on peut l'étendre aux fonctionnelles de $D_{q, 1}(q>1)$, considéré comme partie de l'espace des distributions $D$ ', en appliquant les résultats de Ustunel ([13b], Propositions II2 et IV1):

Lemma 3.3. Soit $F$ élément de $D_{q, 1} q>1$. Alors:

$$
F=E(F)+\sum_{i} \int_{0}^{T} E\left(D_{t}^{i} F / \mathscr{G}_{t}\right) d W_{t}^{i}
$$

où $\mathscr{G}$ est la filtration naturelle engendrée par les applications coordonnées, et l'intégrale est une intégrale de Ito généralisée.

Ce lemme permet de montrer la proposition suivante:

Proposition 3.4. Soit un processus $u$ de $D_{q, 2}(L), q>1$. Alors, l'intégrale de Skorohod peut être représentée par:

$$
\delta(u)=\sum_{i} \int_{0}^{T} E\left(u_{t}^{i}+\int_{0}^{t} D_{t}^{i} u_{s} \delta W_{s} / \mathscr{G}_{t}\right) d W_{t}^{i} .
$$

où l'intégrale est une intégrale de Ito généralisée.

Démonstration. Il suffit d'appliquer (3.21) à la fonctionnelle $\delta(u)$ qui appartient à $D_{q, 1}$ grâce à l'hypothèse, le fait que l'espérance de $\delta(u)$ est nulle et la formule de commutation (3.16). 
4. Enfin, la "formule de Itô" étendue aux processus anticipatifs donnée dans [7] ou [13] nécessite des hypothèses relativement contraignantes d'intégrabilité sur les processus, afin de pouvoir s'appliquer à des fonctions très générales. On peut montrer cette formule simplement pour le carré, en affaiblissant un peu les hypothèses:

Proposition 3.5. Soit un processus $u$ de $D_{2,2}(\boldsymbol{L})$ :

$$
(\delta(u))^{2}=2 \int_{0}^{T} u_{s} \delta_{s} u \delta W_{s}+\int_{0}^{T} u_{s}^{2} d s+2 \int_{0}^{T} u_{s} \int_{0}^{s} D_{s} u_{r} \delta W_{r} d s
$$

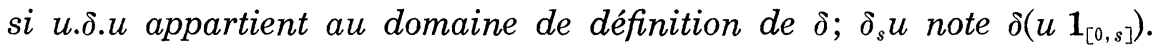

En revanche, pour l'exponentielle, on applique simplement le résultat cité dans [7], en explicitant hypothèses et résultat pour ce cas particulier:

Proposition 3.6. Soit un processus $u$ de $D_{2,2}(L)$ tel que Du appartient $\grave{a} L^{4+\varepsilon}\left([0, T]^{2} \times W\right)$ et $D^{2} u \grave{a} L^{4+\varepsilon}\left([0, T]^{3} \times W\right)$; et soit $v$ un processus de $D_{2,1}(L)$ tel que $D v$ appartient à $L^{4}\left([0, T]^{2} \times W\right)$. Alors, le processus $L$ défini par $L_{t}=\exp \left(\delta_{t}(u)+\int_{0}^{t} v_{s} d s\right)$ vérifie:

$$
L_{t}=1+\int_{0}^{t} L_{s}\left[u_{s} \delta W_{s}+v_{s} d s+1 / 2 u_{s}^{2} d s+u_{s}\left(\int_{0}^{s} D_{s} u_{r} \delta W_{r}+D_{s} v_{r} d r\right) d s\right]
$$

5. Pour conclure ce paragraphe, on applique les résultats précédents pour justifier l'introduction du processus $L^{h}$ défini en (2.15). En effet, sur l'espace produit construit en (2.3), tout ce qui dépend de $X$ peut être considéré comme non aléatoire: $X_{t}$ est un paramètre temporel. Et on considère l'espase de Wiener associé au brownien $B$. Sur cet espace de Wiener, il convient de s'assurer que $Y$ et $H$ sont des éléments de $D_{2,1}(L)$. Or, $Y$ est solution de l'équation différentielle stochastique (1.1) dont les coefficients sont deux fois dérivables à dérivées bornées. En reprenant exactement la démonstration de [3] (p. 337 à 339) (cf. théorème 2.1 de [6]) on obtient immédiatement:

$$
Y_{t} \in D_{2,1} \text { pour tout } t
$$

et comme $Y$ est à valeurs dans $L, Y$ appartient à $D_{2,1}(L)$. Puis, on obtient, par les règles de dérivation, que $D_{s} Y$ est solution de:

$$
D_{s}^{k} Y_{t}^{i}=\mathbf{1}_{[0, t]}(s)\left(\delta_{i k}+\int_{0}^{t} \partial_{j} H_{u}^{i} D_{s}^{k} Y_{u}^{j} d u\right) ; \quad i \text { et } k=1, \cdots, d .
$$


La règle de dérivation (3.17) implique que $H$ est aussi dans $D_{2,1}(L)$ et son gradient stochastique vérifie:

$$
D_{s}^{k} H_{t}^{i}=\mathbf{1}_{[0, t]}(s) \partial_{j} H_{t}^{i}\left(\delta_{i k}+\int_{0}^{t} D_{s}^{k} H_{u}^{j} d u\right)
$$

La bornitude du gradient de $H$ et le lemme de Gronwall montre que $D H$ est uniformément borné sur $\Omega \times[0, T]^{2}$. Comme par ailleurs, les hypothèses donnent que $H$ est dans $L^{2}$, on déduit par la définition (3.9).

$$
H \in D_{21}(L) \text { et } H_{t} \in D_{2,1} \text { pour tout } t \text {. }
$$

Puisque $H$ est deux fois dérivable, on peut itérer la dérivation stochastique sur (3.27); la bornitude des dérivées secondes de $H$ (2.4) montre que $D^{2} H$ est également borné et donc $H$ est de fait dans $D_{2,2}(L)$.

On peut alors appliquer la relation (3.20) au couple $\left(H_{i n}, 1\right)$ sur l'intervalle $I_{i}$ :

$$
\delta\left(H_{i h}^{j} \mathbf{1}_{I_{i}}\right)=H_{i h}^{j}\left(B_{i h}^{j}-B_{(i-1) h}^{j}\right)-\int_{(i-1) h}^{i n} D_{s}^{j} H_{i h}^{j} d s
$$

On déduit par ailleurs de (1.1):

$$
H_{i n}\left(Y_{i h}-Y_{(i-1) h}\right)=H_{i n}\left(B_{i h}-B_{(i-1) h}\right)+\int_{(i-1) h}^{i n} H_{i h} H_{s} d s
$$

Ces deux dernières relations impliquent:

$$
\begin{aligned}
& H_{i h}\left(Y_{i h}-Y_{\left(i-1_{j}\right) h}\right)-h \sum \partial_{j} H_{i n}^{j} \\
& =\int_{I_{i}} H_{s}^{h} \delta B_{s}+\int_{I_{i}}\left(H_{s}^{h} H_{s}+\sum_{j} D_{s}^{j} H_{s}^{h, j}-\sum_{j} \partial_{j} H_{s}^{h, j}\right) d s
\end{aligned}
$$

où $H_{t}^{h}$ et $D_{s} H_{t}^{h}$ désignent les processus constants sur $I_{i}$ et égaux à $H_{i h}$ et $D_{s} H_{i h}$ respectivement. Si l'on reporte cette expression (3.31) dans la définition (1.3) de $L_{N}^{h}$, on obtient:

$$
L_{N}^{h}=\exp \int_{0}^{N h}\left(H_{s}^{h} \delta B_{s}+\left(H_{s}^{h} H_{s}-1 / 2\left\|H_{s}^{h}\right\|^{2}+D_{s} H_{s}^{h}-\operatorname{div} H_{s}^{h}\right) d s\right)
$$

où l'on omet sommations et indices, et l'on vérifie que, effectivement, le processus $L^{h}$ défini en (2.15) coïncide avec $L_{N}^{h}$ au temps $N h$.

L'indépendance de $X$ et de $B$ permet de considérer de la même façon l'espace de Wiener associé au mouvement brownien $V$ qui conduit la diffusion qu'est le signal $X$, à savoir l'espace introduit en (2.2). On notera $D^{\alpha}$ le gradient stochastique et $D_{p, k}^{\alpha}$ les espaces de Sobolev associés aux 
composantes $\left\{V^{\alpha}, \alpha=1 \cdots n\right\}$ du brownien $V$, et plus globalement: $D^{x}$ et $D_{p, k}^{X}$ pour ce qui concerne l'ensemble des composantes. Remarquons que les hypothèses choisies sur $H$ et $X$ montrent que $H$ est un élément de $D_{p, 1}^{X}(L)$ pour tout $p>1$, en adaptant démonstrations et résultats de Taniguchi [16] dont les hypothèses sont plus fortes que dans le cas présent, ou ceux de Davis ([17], corollaire du théorème 3.2); prenons ici plus simplement l'hypothèse supplémentaire:

$$
H_{0} \in D_{p, k}^{X}, \sigma \in D_{p, 2}^{X}, b \in D_{p, k}^{X} \text { pour tout } p>1
$$

ce qui montre immédiatement que $H$ appartient à $D_{p, k}^{X}(L)$ pour tout $p>1$, avec (2.5) et (3.14).

On peut alors montrer le lemme suivant dans le cas $\mathrm{B}$ où $H$ ne dépend que de $X$ :

Lemme 3.7. Sous les hypothèses (2.4) B, (2.5) et (3.33), la variable $L_{T}^{-1}$ définie par $L$ (2.6) est, conditionnellement en $\mathscr{G}_{T}^{Y}$, un élément de $D_{r, 1}^{X}$ pour tout $r>1$, avec:

$$
D_{v}^{X}\left(L_{T}^{-1}\right)=L_{T}^{-1} \int_{v}^{T} D_{v}^{X} H_{s}\left(d Y_{s}-H_{s} d s\right)
$$

Démonstration. Conditionnellement en $\mathscr{G}_{T}^{Y}$, l'expression de $L_{T}^{-1}$ est:

$$
L_{T}^{-1}=\exp \int_{0}^{T}\left(H_{s} d Y_{s}-1 / 2\left\|H_{s}\right\|^{2} d s\right)
$$

Il s'agit d'appliquer le lemme 3.2 et donc de montrer que $L_{T}^{-1}$ appartient à $L^{p}\left(\Omega^{X}\right)$ et que $G=\int_{0}^{T}\left(H_{s}\left(d Y_{s}-1 / 2\left\|H_{s}\right\|^{2} d s\right)\right.$ appartient à $D_{q 1}^{X}$ avec $1 / p$ $+1 / q=1 / r<1$. Pour donner un sens à (3.35) où l'on considère $Y$ comme un paramètre déterministe, on réécrit l'intégrale stochastique à l'aide de la formule classique de Itô et de (2.5):

$$
L_{T}^{-1}=\exp \left[\int_{0}^{T}\left(\left(Y_{T}-Y_{s}\right)\left(\sigma_{s} d V_{s}+b_{s} d s\right)-1 / 2\left\|H_{s}\right\|^{2} d s\right)\right]
$$

On met alors le $p^{\circ}$ moment de $L_{T}^{-1}$ sous forme de produit d'une $\mathscr{G}_{T}^{X}$ martingale par un coefficient positif, borné conditionnellement en $Y$ :

$$
\begin{aligned}
\left(L_{T}^{-1}\right)^{p}= & \exp \left[\int_{0}^{T}\left(p\left(Y_{T}-Y_{s}\right) \sigma_{s} d V_{s}-1 / 2\left\|p\left(Y_{T}-Y_{s}\right) \sigma_{s}\right\|^{2} d s\right)\right] \\
& \times \exp \int_{0}^{T}\left(1 / 2\left\|p\left(Y_{T}-Y_{s}\right) \sigma_{s}\right\|^{2}+p\left(Y_{T}-Y_{s}\right) b_{s}-p / 2\left\|H_{s}\right\|^{2}\right) d s
\end{aligned}
$$


qui est donc intégrable sur $\left(\Omega^{x}, \boldsymbol{P}^{X}\right)$ pour tout $p$.

On montre ensuite que l'appartenance de $H$ à $D_{q 1}^{X}$ pour tout $q>1$ implique celle de $1 / 2 \int_{0}^{T}\left\|H_{s}\right\|^{2} d s$ à $D_{q 1}$ pour tout $q>1$. Il suffit pour cela d'appliquer le critère (3.10): $H$ est limite d'une suite d'éléments $u_{n}$ de $S^{L}$, de Cauchy pour la norme \|\|$_{p, 1}^{L}$; alors $1 / 2 \int_{0}^{T}\left\|u_{n, s}\right\|^{2}$ ds est clairement une suite de $S$ dont on montre qu'elle est de Cauchy pour la norme \|\|$_{p, 1}$. Par passage à la limite, on obtient de plus:

$$
D_{v}^{X} 1 / 2 \int_{0}^{T}\left\|H_{s}\right\|^{2} d s=\int_{0}^{T} D_{v}^{X} H_{s} H_{s} d s .
$$

On étudie enfin le terme $\int_{0}^{T}\left(Y_{T}-Y_{s}\right)\left(\sigma_{s} d V_{s}+b_{s} d s\right)$. L'hypothèse (3.33) montre que ce terme appartient à $D_{q 1}^{X}$ pour tout $q$ et les règles de dérivation permettent d'obtenir;

$$
\begin{aligned}
& D_{v}^{X} \int_{0}^{T}\left(Y_{T}-Y_{s}\right)\left(\sigma_{s} d V_{s}+b_{s} d s\right) \\
& \quad=\left(Y_{T}-Y_{v}\right) \sigma_{v}+\int_{v}^{T}\left(Y_{T}-Y_{s}\right)\left(D_{v}^{X} \sigma_{s} d V_{s}+D_{v}^{X} b_{s} d s\right)
\end{aligned}
$$

utilisant en outre que le gradient stochastique $D_{v} u_{s}$ est nul si $s$ est inférieur à $v$ lorsque $u$ est adapté (voir (3.5) par exemple...)

Ceci étant, une simple identification permet de remplacer (3.39) par $\int_{0}^{T} D_{v}^{X} H_{s} d Y_{s}$, en utilisant la formule de Itô comme en (3.36), ce qui achève la démonstration du lemme.

\section{§IV. Estimation de l'erreur}

Il s'agit maintenant d'obtenir un ordre de grandeur en moyenne quadratique de l'erreur commise en utilisant $\pi^{h}(Z)$ (2.14) comme filtre à la place de $\pi_{T}(Z)$ (2.13). L'objet de cette partie est donc de montrer:

Proposition 4.1. Soit le système de filtrage dont l'observation est définie en (1.1), où le "bruit" est indépendant du signal, et la fonction du signal vérifie les hypothèses (2.4) et (2.5). Alors, sous l'hypothèse A de (2.4), pour $Z$ variable $\mathscr{G}_{T}^{X}$-mesurable et bornée:

$$
\left\|\pi^{h}(Z)-\pi_{T}(Z)\right\|_{2}=0(\sqrt{h})
$$

Sous l'hypothèse $\mathrm{B}$ de (2.4), pour $Z$ variable $\mathscr{G}_{T}^{X}$-mesurable et bornée de $D_{p, 1}^{X}$ et si $H$ vérifie (3.33): 


$$
\left\|\pi^{h}(Z)-\pi_{T}(Z)\right\|_{2}=0(h)
$$

Démonstration. On peut supposer, sans perte de généralité, que le pas de discrétisation $h$ est exactement $T / N, N$ étant le nombre d'observations discrètes. Pour tout $h$, on introduit le processus $A^{h}$, produit de $L^{h}$ et $L$, c'est-à-dire:

$$
A_{t}^{h}=\exp \left[\delta_{t}\left(H^{h}-H\right)+\int_{0}^{t}\left(D_{s} H_{s}^{h}-\operatorname{div} H_{s}^{h}-1 / 2\left\|H_{s}^{h}-H_{s}\right\|^{2}\right) d s\right]
$$

où comme en (2.15), on omet sommations et indices. Sauf mention contraire, $D, \delta, D_{p, k}$ désignent dans ce paragraphe opérateurs et espaces afférents à l'espace de Wiener $(W, \mu)$ donné en (2.1).

La démonstration s'appuie sur l'identité suivante (voir [10] par exemple):

$$
\pi^{h}(Z)-\pi_{T}(Z)=E_{Q}\left[\left(A_{T}^{h}-1\right)\left(Z-\pi^{h}(Z)\right) / \mathscr{G}_{T}^{Y}\right]
$$

qui permet d'évaluer l'erreur en fonction la norme quadratique de $A_{T}^{h}-1$, puisque $\left(Z-\pi^{h}(Z)\right)$ est borné; (4.4) se montre simplement en développant le membre de droite, on utilise (2.13) et (2.14) et on identifie.

Le lemme suivant, clé de la mesure de l'approximation, servira à plusieurs reprises:

Lemme 4.2. Pour tout réel $q \geq 1$, le processus $H^{h}-H$ vérifie:

$$
\left\|\int_{0}^{T}\right\| H_{t}^{h}-H_{t}\left\|^{2} d t\right\|_{q} \leq c_{q} h
$$

où $c_{q}$ est une constante.

Démonstration. Sous l'hypothèse (2.4), le résultat est classique pour $q=1$. Soit donc $q>1$. On tire de (2.4), lorsque $t$ appartient à l'intervalle $I_{i}$ :

$$
H_{t}^{h}-H_{t}=\int_{t}^{i n}\left(b_{s} d s+\sigma_{s} d \Omega_{s}\right)
$$

d'où il vient:

$$
\left\|H_{t}^{h}-H_{t}\right\|^{2} \leq 2\left(\int_{t}^{i n} b_{s} d s\right)^{2}+2\left(\int_{t}^{i n} \sigma_{s} d \Omega_{s}\right)^{2}
$$

Le permier terme est inférieur à $2 h \int_{I_{i}}\left|b_{s}\right|^{2} d s$ dont l'intégrale sur $[0, T]$ se majore de la façon suivante: 


$$
\sum_{i} \int_{I_{i}} 2\left(\int_{t}^{i h} b_{s} d s\right)^{2} d t \leq 2 h^{2} \int_{0}^{T}\left|b_{s}\right|^{2} d s
$$

Puisque $b$ est dans $L^{q}(\Omega \times[0, T])$ pour tout $q$, la $q$-norme de (4.8) est majorée par $2 h^{2}\left\|\int_{0}^{T}\left|b_{s}\right|^{2} d s\right\|_{q}$.

On évalue ensuite la $q^{\circ}$ puissance du second terme à droite de (4.7), on note $c(t)$ le temps ih lorsque $t$ appartient à $I_{i}$ et on utilise l'inégalité de Hölder:

$$
\left(\int_{0}^{T}\left(\int_{t}^{c(t)} \sigma_{s} d \Omega_{s}\right)^{2} d t\right)^{q} \leq T^{q-1} \int_{0}^{T}\left(\int_{t}^{c(t)} \sigma_{s} d \Omega_{s}\right)^{2 q} d t
$$

Fixant $t$, on applique l'inégalité de Burkholder à la martingale $\int_{t} \sigma_{s} d \Omega_{s}$ ([1] 2 p. 304):

$$
E\left(\int_{t}^{c(t)} \sigma_{s} d \Omega_{s}\right)^{2 q} \leq(8 q)^{2 q} E\left(\int_{t}^{c(t)}\left|\sigma_{s}\right|^{2} d s\right)^{q}
$$

Or $\left|\sigma_{s}\right|$ est majoré par $\|\sigma\|_{\infty}$ :

$$
\left(\int_{t}^{c(t)}\left|\sigma_{s}\right|^{2} d s\right)^{q} \leq(c(t)-t)^{q}\|\sigma\|_{\infty}^{2 q}
$$

et l'on déduit une majoration de l'espérance de (4.9):

$$
\left\|\int_{0}^{T}\left(\int_{t}^{c(t)} \sigma_{s} d \Omega_{s}\right)^{2} d t\right\|_{q} \leq T h(8 q)^{2}\left(E\left(\|\sigma\|_{\infty}^{2 q}\right)\right)^{1 / q}
$$

ce qui montre (4.5) avec (4.7) et (4.8).

Si l'on reprend (4.6) en fixant $t$, on peut.montrer sous les mêmes hypothèses et avec les mêmes calculs:

$$
\forall q \geq 1, H^{h}-H \in L^{q}([0, T] \times \Omega) \text { et } \forall t, H_{t}^{h}-H_{t} \in L^{q}
$$

Cette remarque et le lemme 4.2 permettent de montrer l'appartenance du processus $H^{h}-H$ aux espaces $D_{p, k}$, ce qui permettra de lui appliquer les résultats de la partie III.

Lemma 4.3. Sous les hypothèses (2.4) et (2.5), le processus $H^{h}-H$ appartient à $D_{q, 2}(L)$, pour tout $q \geq 2$. Si de plus $H$ est indépendant $d u$ brownien $B$ (hypothèse $\mathrm{B}$ ), $H^{h}-H$ appartient $\grave{a} D_{q, k}(L)$, pour tout $q$ et entier $k$.

Démonstration. On a vu en fin de troisième partie que $H$ est un élément de $D_{2,2}(L)$ et que $D H$ et $D^{2} H$ sont bornés; il en est donc de même 
du processus $H^{h}-H$ qui est de plus dans $L^{2 q}(L)$ pour tout $q \geq 1$ grâce à (2.4): la caractérisation (3.9) de $D_{q, 2}(L)$ montre le premier résultat. Le second est une évidence: sous l'hypothèse $\mathrm{B}$, div $H$ est nul donc aussi $D H$ et tous les gradients stochastiques $D^{k} H$ !

Ceci permet d'appliquer la "formule de Itô" au processus $A^{h}$ et d'obtenir:

Lemme 4.4. Le processus $A^{h}$ défini en (4.3) vérifie:

$$
A_{T}^{h}-1=\delta\left(A^{h}\left(H^{h}-H\right)\right)+\int_{0}^{T} A_{t}^{h}\left[\left(D_{t} H_{t}^{h}-\operatorname{div} H_{t}^{h}\right)+\left(H_{t}^{h}-H_{t}\right) M_{t}^{h}\right] d t
$$

avec le processus vectoriel $M^{h}$ :

$$
M_{t}^{h}=\int_{h[t / h]}^{t}\left[D_{t} H_{s}^{h} \delta B_{s}+\left(D_{t}\left(D_{s} H_{s}^{h}-\operatorname{div} H_{s}^{h}\right)-D_{t} H_{s}^{h}\left(H_{s}^{h}-H_{s}\right)\right) d s\right]
$$

où $[t / h]$ désigne la partie entière de $t / h, H^{h}$ et $\operatorname{div} H^{h}$ les processus constants égaux à $H\left(X_{i h}, Y_{i h}\right)$ et $\operatorname{div}_{y} H\left(X_{i h}, Y_{i h}\right)$ sur $I_{i}$.

(Comme en (2.15) et (3.32), sommations et indices de $H$ sont omis).

Démonstration. On applique simplement la proposition 3.6 à:

$$
u_{t}=H_{t}^{h}-H_{t}, \quad v_{t}=D_{t} H_{t}^{h}-\operatorname{div} H_{t}^{h}-1 / 2\left\|H_{t}^{h}-H_{t}\right\|^{2}
$$

On vient de voir que $u$ appartient à $D_{2,2}(L)$ et que Du et $D^{2} u$ sont bornés. De plus, $v$ est un élément de $D_{q, 1}(L)$ pour tout $q$ : c'est la somme de processus bornés admettant un gradient stochastique borné et de $\|u\|^{2}$ qui appartient à $D_{q, 2}(L)(q>1)$ grâce au lemme 3.1. Evaluons maintenant $D v$ en utilisant les règles de dérivation; si $t$ appartient à $I_{i}$ :

$$
\begin{aligned}
D_{s} v_{t}=D_{s} D_{t} H_{t}^{h}-\mathbf{1}_{[0, i h]}(s) \partial^{2} H_{t}^{h} & \left(1+\int_{0}^{i n} D_{s} H_{u} d u\right) \\
& -\left(D_{s} H_{t}^{h}-D_{s} H_{t}\right)\left(H_{t}^{h}-H_{t}\right) .
\end{aligned}
$$

Les termes de cette somme sont tous bornés, sauf le dernier qui est un élément de $L^{q}\left([0, T]^{2} \times \Omega\right)$ pour tout $q$; il en est donc de même pour $D v$. Les hypothèses de la proposition 3.6 sont vérifiées et on obtient (4.14) et (4.15) après avoir, comme en (3.39), utilisé l'adaptabilité de $H$ qui annulle le gradient stochastique $D_{t} H_{s}$ lorsque $s<t$.

Pour évaluer la norme de $A_{T}^{h}-1$, on va donc étudier la norme de chacun des termes de son développement, et comme c'est classique en calcul de Itô, on décompose l'intégrale $\delta\left(A^{h}\left(H^{h}-H\right)\right)$ : 


$$
\delta\left(A^{h}\left(H^{h}-H\right)\right)=\delta\left(H^{h}-H\right)+\delta\left(\left(A^{h}-1\right)\left(H^{h}-H\right)\right) .
$$

Pour traiter le deuxième terme ci-dessus, on utilise l'existence de moments de $A^{h}$, uniformément bornés en $h$ :

Lemme 4.5. Sous les hypothèses (2.4) (2.5), le processus $A^{h}$ défini en (4.3) vérifie:

Il existe $p>2$ et $h_{0}>0$ tels que $\forall h \leq h_{0}, \forall t \in[O, T],\left\|A_{t}^{h}\right\|_{p} \leq M$.

Démonstration. Par définition, le processus $A^{h}$ s'écrit:

$$
A_{t}^{h}=\exp \delta_{t}\left(H^{h}-H\right) \exp \int_{0}^{t} v_{s} d s
$$

a) Sous l'hypothèse (2.4) $\mathrm{A}$, le processus $v$, et donc le deuxième facteur, est uniformément borné sur $\Omega \times[0, T]$. On peut décomposer le premier facteur en le produit suivant:

$$
\begin{aligned}
& \exp \delta_{t}\left(H^{h}-H\right) \\
& \quad=\exp \sum \Delta_{i} H \Delta_{i} B \exp \int_{0}^{t}\left(\bar{H}_{s}-H_{s}\right) d B_{s} \exp \int_{0}^{t}-D_{s} H_{s}^{h} d s
\end{aligned}
$$

où la sommation $\sum$ s'entend pour $(i-1) h \leq t$ et $\bar{H}_{s}$ est égal à $H_{(i-1) h}$ sur $I_{i}$. Le troisième facteur de ce produit est uniformément borné. Le second, au coefficient borné $\exp -1 / 2 \int_{0}^{t}\left\|\bar{H}_{s}-H_{s}\right\|^{2} d s$ près, est une martingale appartenant à tout $L^{q}$. Pour traiter le premier facteur, on majore le produit des accroissements de $H$ et $B$ par la demi-somme de leur carré:

$$
2 \sum \Delta_{i} H_{i} \Delta_{i} B \leq \sum\left\|\Delta_{i} H\right\|^{2}+\sum\left\|\Delta_{i} B\right\|^{2}
$$

On a donc à étudier l'intégrabjlité du produit suivant:

$$
\left(P^{h}\right)^{p}=\exp p / 2 \sum\left\|\Delta_{i} H\right\|^{2} \exp p / 2 \sum\left\|\Delta_{i} B\right\|^{2} \exp p \int_{0}^{\iota}\left(\bar{H}_{s}-H_{s}\right) d B_{s}
$$

On déduit par une inégalité de Hölder avec $1 / q+1 / r+1 / u=1$ :

$$
\begin{aligned}
&\left\|P^{h}\right\|_{p} \leq\left\|\exp 1 / 2 \sum\right\| \Delta_{i} H\left\|^{2}\right\|_{p q}\left\|\exp 1 / 2 \sum\right\| \Delta_{i} B\left\|^{2}\right\|_{p r} \\
& \times\left\|\exp \int_{0}^{t}\left(\bar{H}_{s}-H_{s}\right) d B_{s}\right\|_{p u}
\end{aligned}
$$

Parce que $H$ est uniformément borné, le troisième facteur est borné uniformément en $t$ et $h$. Le deuxième facteur est la norme du produit 
de $N d$ facteurs indépendants, soit $\exp \operatorname{pr} / 2\left|\Delta_{i} B^{j}\right|^{2}$ pour $i=1 \cdots N$ et $j=1$ $\cdots d$, dont on calcule sans peine l'espérance, connaissant la loi de l'accroissement du brownien:

$$
\begin{aligned}
& E\left(\exp (\operatorname{pr} / 2)(\Delta B)^{2}\right) \\
& \quad=1 / \sqrt{2 \pi} \int \exp \left(h p r x^{2} / 2\right) \exp \left(-x^{2} / 2\right) d x=(1-h p r)^{-1 / 2}
\end{aligned}
$$

à condition que $h$ soit assez petit $(h<1 / p r)$. Le deuxième facteur vaut donc:

$$
\left\|\exp 1 / 2 \sum\right\| \Delta_{i} B\left\|^{2}\right\|_{p r}=(1-h p r)^{-T d / 2 h p r}
$$

qui est uniformément borné pour $h$ assez petit.

Enfin, exprimons le carré de l'accroissement de $H$ par la formule de Itô:

$$
\left\|\Delta_{i} H\right\|^{2}=\int_{I_{i}} 2\left(H_{s}-\bar{H}_{s}\right)\left(b_{s} d s+\sigma_{s} d W_{s}\right)-\left|\sigma_{s}\right|^{2} d s
$$

On a donc à calculer l'espérance de l'exponentielle suivante:

$$
\begin{aligned}
& \exp p q \int_{0}^{t}\left[\left(H_{s}-\bar{H}_{s}\right)\left(b_{s} d s+\sigma_{s} d W_{s}\right)+1 / 2\left|\sigma_{s}\right|^{2} d s\right] \\
& =\exp (p q) \int_{0}^{t}\left[\left(H_{s}-\bar{H}_{s}\right) \sigma_{s} d W_{s}-1 / 2 p^{2} q^{2} \int_{0}^{t}\left\|\left(H_{s}-\bar{H}_{s}\right) \sigma_{s}\right\|^{2} d s\right] \\
& \quad \times \exp \int_{0}^{t}\left(1 / 2 p^{2} q^{2}\left\|\left(H_{s}-\bar{H}_{s}\right) \sigma_{s}\right\|^{2}+p q\left(H_{s}-\bar{H}_{s}\right) b_{s}+p q / 2\left\|\sigma_{s}\right\|^{2}\right) d s
\end{aligned}
$$

Le premier facteur à droite de (4.28) est une martingale exponentielle (sous les hypothèses choisies). L'hypothèse (2.4) A montre que $b$ et $\sigma$ sont bornés, donc le deuxième facteur à droite de (4.28) est borné, ce qui conclut le cas A avec (4.24) et (4.26).

b) Sous l'hypothèse (2.4) $\mathrm{B}$, on déduit de (4.14)

$$
A_{t}^{h}-1=\delta_{t}\left(A^{h}\left(H^{h}-H\right)\right)
$$

Il est clair que la $\mathscr{G}_{T}^{X}$-espérance conditionnelle d'une intégrale de Skorohod sur $\left(W, \mathscr{G}^{B}, \mu\right)$ est nulle: il suffit d'appliquer la formule d'intégration par partie à une fonctionnelle $F \mathscr{G}_{T}^{X}$-mesurable et bornée, donc de $B$-gradient nul à cause de l'indépendance entre $X$ et $B$ :

$$
E(F \delta((u))=E(\langle D F, u\rangle)=0
$$

Ainsi obtient-on pour tout $t$ : 


$$
E\left(A_{t}^{h} / \mathscr{G}_{T}^{X}\right)=1
$$

Notant alors $A_{p, t}^{h}$ le processus obtenu comme $A^{h}$, mais avec $p H$ au lieu de $H$, on obtient grâce à la définition (4.3):

$$
\left(A_{t}^{h}\right)^{p}=A_{p, t}^{h} \exp \left(p^{2}-p\right) / 2 \int_{0}^{t}\left\|H_{s}^{h}-H_{s}\right\|^{2} d s
$$

On tire de (4.31) et (4.32):

$$
E\left[\left(A_{t}^{h}\right)^{p} / \mathscr{G}_{T}^{X}\right]=\exp \left(p^{2}-p\right) / 2 \int_{0}^{t}\left\|H_{s}^{h}-H_{s}\right\|^{2} d s
$$

puisqu'ici $H$ est $\mathscr{G}_{T}^{X}$-mesurable. Sous la condition $\mathrm{B}, b$ est borné comme $\sigma$; (4.7) (4.8) donnent alors la majoration:

$$
\int_{0}^{t}\left\|H_{s}^{h}-H_{s}\right\|^{2} d s \leq M h^{2}+2 \int_{0}^{T}\left\|\int_{s}^{c(s)} \sigma_{u} d V_{u}\right\|^{2} d s
$$

Ceci permet de majorer (4.33), posant $\rho=p^{2}-p, p$ étant fixé $>2$ :

$$
E\left[\left(A_{t}^{h}\right)^{p} / G_{T}^{X}\right] \leq K \exp \rho \int_{0}^{T}\left\|\int_{s}^{c(s)} \sigma_{u} d V_{u}\right\|^{2} d s
$$

dont l'espérance est celle d'un produit que l'on majore par une inégalité de Hölder:

$$
E\left[\left(A_{t}^{h}\right)^{p}\right] \leq K \prod_{i=1, N}\left\|\exp \rho \int_{I_{i}}\right\| \int_{s}^{c(s)} \sigma_{u} d V_{u}\left\|^{2} d s\right\|_{N}
$$

Chacun des $N$ facteurs est la racine $N^{\circ}$ de l'espérance d'une série entière dont on majore chacun des termes:

$$
\left(\rho N \int_{I_{i}}\left\|\int_{s}^{c(s)} \sigma_{u} d V_{u}\right\|^{2} d s\right)^{r} / r ! \leq(\rho N)^{r} h^{r-1} \int_{I_{i}}\left\|\int_{s}^{c(s)} \sigma_{u} d V_{u}\right\|^{2 r} d s / r !
$$

L'inégalité de Burkholder donne une majoration trop forte et l'on obtient une série non convergente. Mais la bornitude de $\sigma$ permet d'évaluer au plus juste l'espérance de $\left\|\int_{s}^{c(s)} \sigma_{u} d V_{u}\right\|^{2 r}$ par récurrence. En effet, fixant $s$ et notant $M_{r}(u)$ l'espérance de $\left\|\int_{s}^{u} \sigma_{u} d V_{u}\right\|^{2 r}$, on obtient par la formule de Itô:

$$
\begin{aligned}
M_{r}(c(s)) & =r(2 r-1) \int_{s}^{c(s)} E\left(\left\|\sigma_{u}\right\|^{2}\left\|\int_{s}^{u} \sigma_{v} d V_{v}\right\|^{2 r-2}\right) d u \\
& \leq r(2 r-1)\|\sigma\|_{\infty}^{2} \int_{s}^{c(s)} M_{r-1}(u) d u .
\end{aligned}
$$

En itérant cette majoration, on obtient finalement: 


$$
M_{r}(c(s)) \leq(2 r-1) ! !\|\sigma\|_{\infty}^{2 r}(c(s)-s)^{r}
$$

L'espérance de la série de terme général (4.37) admet donc la majoration:

$$
E\left(\exp \left(\rho N \int_{I_{i}}\left\|\int_{s}^{c(s)} \sigma_{u} d V_{u}\right\|^{2} d s\right)\right) \leq \sum_{r \geq 0}\left(\rho N h^{2}\|\sigma\|_{\infty}^{2}\right)^{n}(2 r-1) ! ! / r !
$$

Puisque $N h=T$, c'est une série entière dont le rayon de convergence vaut $1 / 2$ et donc (4.40) est définie dès que $h$ est assez petit et admet de plus un majorant indépendant de $i=1 \cdots N$. On se restreint alors à cet $h$ assez petit pour que le produit des $N$ racines $N^{\circ}$ des facteurs (4.40), soit (4.36), soit défini et admette une majoration uniforme, ce qui montre que le $p^{\circ}$ moment de $A_{t}^{h}$ est uniformément majoré en $h$ et $t$, et achève la démonstration de (4.19) dans le cas B.

Ceci étant, on montre l'appartenance de $A^{h}$ aux espaces $D_{p, k}(\boldsymbol{L})$.

Lemme 4.6. Le processus $A^{h}$ défini en (4.3) appartient à $D_{p, 1}(L)$ sous l'hypothèse $A$ et à $D_{p, 2}(L)$ sous l'hypothèse $\mathrm{B}$, pour un $p>2$.

Démonstration. Le processus $A^{h}$ est l'exponentielle, fonction de classe $C^{\infty}$, de la fonctionnelle $\delta\left(H^{h}-H\right)+\int v_{s} d s$ défini en (4.16). Or, le lemme 4.3 et la règle de commutation permettent de dériver stochastiquement deux fois $\delta\left(H^{h}-H\right)$, et on obtient:

$$
D_{s} D_{t} \delta\left(H^{h}-H\right)=D_{s}\left(H_{t}^{h}-H_{t}\right)+D_{t}\left(H_{s}^{h}-H_{s}\right)+\delta\left(D_{s} D_{t}\left(H^{h}-H\right)\right)
$$

dont les deux premiers termes sont bornés, et le troisième est l'intégrale de Skorohod d'une fonctionnelle bornée: $\delta\left(H^{h}-H\right)$ appartient donc à $D_{q, 2}(L)$ pour tout $q$. On a vu par ailleurs dans la démonstration du lemme 4.4 que $v$ appartient à $D_{q, 1}(L)$ pour tout $q$; il en donc de même pour son intégrale. Par ailleurs, on vient de montrer que $A_{t}^{h}$ admet un moment d'ordre $p, p>2$. Le lemme 3.2 montre alors que $A^{h}$ appartient à $D_{p^{\prime}}(\boldsymbol{L})\left(p^{\prime}>p\right)$ avec pour vecteur gradient:

$$
D_{s}^{i} A_{t}^{h}=A_{t}^{h}\left(H_{s}^{h, i}-H_{s}^{i}+\delta_{t}\left(D_{s}^{i}\left(H^{h}-H\right)\right)+\int_{0}^{t} D_{s}^{\imath} v_{r} d r\right)
$$

Si de plus l'hypothèse $B$ est vérifiée, l'expression ci-dəssus se simplifie notablement du fait que $D H$ est nul:

$$
D_{s}^{i} A_{t}^{h}=A_{t}^{h}\left(H_{s}^{h, i}-H_{s}^{i}\right)
$$

c est-à-dire le produit d'un élément de $D_{p^{\prime}, 1}(L)$ par $H^{h}-H$ qui appartient 
MONIQUE PONTIER

à $D_{q, 1}(L)$ pour tout $q$; le lemme 3.1 montre que $D A^{h}$ appartient à $D_{p^{\prime \prime}, 1}$ $\left(p^{\prime \prime}<p^{\prime}\right)$ et donc $A^{h}$ est un élément de $D_{p, 2}(L)$ pour un $p>2$. On a de plus dans ce cas la matrice gradient stochastique d'ordre 2:

$$
D_{r}^{i} D_{s}^{j} A_{t}^{h}=A_{t}^{h}\left(H_{s}^{h, i}-H_{s}^{i}\right)\left(H_{r}^{h, i}-H_{r}^{i}\right)
$$

Démonstration de 4.1. On est ici sous l'hypothèse où $H$ est fonction de l'observation $Y$. On tire de (4.14):

$$
\begin{aligned}
& \left\|A_{T}^{h}-1\right\| \leq\left\|\delta\left(H^{h}-H\right)\right\|+\left\|\delta\left(A_{T}^{h}-1\right)\left(H^{h}-H\right)\right\| \\
& \quad+\left\|\int_{0}^{T} A_{t}^{h}\left(D_{t} H_{t}^{h}-\operatorname{div} H_{t}^{h}\right) d t\right\|+\left\|\int_{0}^{T} A_{t}^{h}\left(H^{h}-H_{t}\right) M_{t} d t\right\|
\end{aligned}
$$

La relation (3.27) montre, si $t$ appartient à $I_{i}$ :

$$
D_{t} H_{t}^{h}-\operatorname{div} H_{t}^{h}=\sum \partial_{j} H_{i n} \int_{t}^{i h} D_{t} H_{u}^{\jmath} d u
$$

La bornitude de $D H$ et $\partial H$ montre que (4.46) est uniformément majoré par $K h$ ( $K$ constante); cette remarque et le lemme 4.5 impliquent:

$$
\left\|\int_{0}^{T} A_{t}^{h}\left(D_{t} H_{t}^{h}-\operatorname{div} H_{t}^{h}\right) d t\right\|_{2} \leq K h\left\|\int_{0}^{T} A_{t}^{h} d t\right\|_{2}=0(h)
$$

On étudie ensuite le processus $M$ (4.15). Son premier terme, si $t$ est dans l'intervalle $I_{i}$, est l'intégrale de Skorohod de $D_{t} H_{i h}$ sur l'intervalle $[(i-1) h, t]$ : c'est donc un $0(\sqrt{h})$ dans tout $L^{q}$. Les termes suivants sont l'intégrale de processus bornés sur ce même intervalle: ils sont uniformément majorés par $K h$, et il vient:

$$
\left\|M_{t}\right\|_{q}=O(\sqrt{h}) \text { pour tout } q .
$$

On majore alors le quatrième terme à droite de (4.45):

$$
\begin{aligned}
& \left(\int_{0}^{T} A_{t}^{h}\left(H_{t}^{h}-H_{t}\right) M_{t} d t\right)^{2} \\
& \quad \leq \int_{0}^{T}\left\|H_{t}^{h}-H_{t}\right\|^{2} d t\left(\int_{0}^{T}\left(A_{t}^{h}\right)^{p} d t\right)^{2 / p}\left(\int_{0}^{T}\left\|M_{t}\right\|^{q} d t\right)^{2 / q}
\end{aligned}
$$

avec $1 / p+1 / q=1 / 2$. On applique une inégalité de Hölder à cette majoration afin d'obtenir:

$$
\begin{aligned}
& E\left(\int_{0}^{T} A_{t}^{h}\left(H_{t}^{h}-H_{t}\right) M_{t} d t\right)^{2} \\
& \quad \leq\left\|\int_{0}^{T}\right\| H_{t}^{h}-H_{t}\left\|^{2} d t\right\|_{q}\left\|\int_{0}^{T}\left(A_{t}^{h}\right)^{p} d t\right\|_{p / 2}^{2 / p}\left\|\left(\int_{0}^{T}\left\|M_{t}\right\|^{q} d t\right)^{2 / q}\right\|_{q}
\end{aligned}
$$


où $p$ (dans (4.49) et (4.50)) est celui du lemme 4.5, ce qui permet, comptetenu également de (4.48) et (4.5), d'obtenir:

$$
\left\|\left(\int_{0}^{T} A_{t}^{h}\left(H_{t}^{h}-H_{t}\right) M_{t} d t\right)^{2}\right\|_{2}=0(h)
$$

Passons à l'étude du terme $\delta\left(\left(A^{h}-1\right)\left(H^{h}-H\right)\right)$. Les lemmes 4.3 et 4.6 (avec le lemme 3.1), montrent que le processus $\left(A^{h}-1\right)\left(H^{h}-H\right.$ ) appartient à $D_{2,1}(L)$ et son gradient stochastique appartient à $L^{2}\left([0, T]^{2} \times \Omega\right)$. On peut donc appliquer la relation d'isométrie ([8], Proposition 3.1):

$$
\begin{aligned}
& E\left[\delta\left(\left(A^{h}-1\right)\left(H^{h}-H\right)\right)\right]^{2}=\int_{0}^{T} E\left[\left(A_{t}^{h}-1\right)^{2}\left\|H_{t}^{h}-H_{t}\right\|^{2}\right] d t \\
& \quad+\iint E\left[D_{s}\left(A_{t}^{h}-1\right)\left(H_{t}^{h}-H_{t}\right) D_{t}\left(A_{s}^{h}-1\right)\left(H_{s}^{h}-H_{s}\right)\right] d s d t
\end{aligned}
$$

La double intégrale est la somme des $N$ intégrales sur $I_{i} \times I_{i}$ de J'intégrande, qui est nul en dehors de ce domaine à cause de la $\mathscr{G}_{i h}$-mesurabilité de $\left(A^{h}-1\right)\left(H^{h}-H\right)$ sur $I_{i}$. De plus, à cause des différentes hypothèses de bornitude, $D_{s}\left(A_{t}^{h}-1\right)\left(H_{t}^{h}-H_{t}\right)$ est majorée par une variable du type $a+b A_{t}^{h}+c A_{t}^{h} \delta_{t}\left(D_{s}\left(H^{h}-H\right)\right)$ dont on peut majorer l'espérance du carré indépendamment de $s$ et $t$. Ainsi, cette double intégrale est-elle un $0(h)$ et puisque $H$ est borné, on tire de (4.52):

$$
E\left[\delta\left(A^{h}-1\right)\left(H^{h}-H\right)\right]^{2} \leq K \int_{0}^{T} E\left(A_{t}^{h}-1\right)^{2} d t+0(h)
$$

On utilise enfin le lemme:

LEMmE 4.7. Sous les hypothèses (2.4) (2.5), la norme quadratique de $\delta\left(H^{h}-H\right)$ est un $0(\sqrt{h})$.

Démonstration. C'est en fait la même que celle de (4.53), en plus simple, car $D\left(H^{h}-H\right)$ est borné, En effet, le processus $H^{h}-H$ étant élément de $D_{q, 1}$, on peut lui appliquer la relation d'isométrie (cf. 4.52):

$$
\begin{aligned}
& E\left[\delta\left(H^{h}-H\right)\right]^{2} \\
& \quad=\int_{0}^{T} E\left\|H^{h}-H\right\|^{2} d t+\iint E\left[D_{s}\left(H_{t}^{h}-H_{t}\right) D_{s}\left(H_{s}^{h}-H_{s}\right)\right] d s d t
\end{aligned}
$$

Le premier terme est un $0(h)$ (lemme 4.2); le second est la somme de $N$ intégrales sur $I_{i} \times I_{i}$, à cause de la $\mathscr{G}_{i n}$-mesurabilité de $H^{h}-H$ sur $[0, i h]$, d'intégrande bornée: $D H$ est borné uniformément sur $[0, T]^{2}$; le 
deuxième terme de (4.54) est donc aussi un $0(h)$ ce qui montre le lemme.

Ce lemme, (4.53), (4.51), (4.47) et (4.45) impliquent:

$$
E\left(A_{T}^{h}-1\right)^{2} \leq 0(h)+K \int_{0}^{T} E\left(A_{t}^{h}-1\right)^{2} d t
$$

ce qui montre (4.1), à ]'aide du lemme de Gronwall, puis de (4.4).

Démonstration de 4.2. On ne suppose plus que $H$ est bornée, mais $H$ est fonction uniquement du signal; alors $\partial H$ et $D H$ sont nuls et la relation (4.14) est réduite à:

$$
A_{t}^{h}-1=\delta_{t}\left(H^{h}-H\right)+\delta_{t}\left(\left(A^{h}-1\right)\left(H^{h}-H\right)\right) .
$$

Le premier terme est un $0(\sqrt{h})$ : c'est en fait l'intégrale de Itô ordinaire du processus "déterministe" $H^{n}-H$, grâce à l'indépendance entre $H$ et $B$.

Néanmoins, suivant [10] mais par une tout autre méthode, on peut montrer, pour $Z$ variable $\mathscr{G}_{T}^{X}$-mesurable et bornée:

$$
\left\|E_{Q}\left(Z \delta\left(H^{h}-H\right) / \mathscr{G}_{T}^{Y}\right)\right\|_{2}=0(h)
$$

La démonstration de (4.57) comporte plusieurs étapes. En premier lieu, on obtient, du moins formellement:

$$
E_{Q}\left[Z \delta\left(H^{h}-H\right) / \mathscr{G}_{T}^{Y}\right]=E_{Q}\left[\int_{0}^{T} E_{Q}\left[Z\left(H_{t}^{h}-H_{t}\right) / \mathscr{G}_{T}^{Y}\right] \delta B_{t} / \mathscr{G}_{T}^{Y}\right]
$$

en calculant l'espérance du produit de chacun des deux membres de cette égalité avec une fonctionnelle $F$ bornée et $\mathscr{G}_{T}^{Y}$-mesurable, par exemple de la forme: $F=f\left(Y\left(t_{1}\right) \cdots Y\left(t_{n}\right)\right)$ avec $f \in C_{b}^{\infty}\left(\boldsymbol{R}^{n}\right)$, dont on remarquera que le gradient stochastique $D_{t} F$ est $\mathscr{G}_{T}^{Y}$-mesurable grâce aux règles de dérivation et au fait que $D_{t} Y_{s}$ est déterministe sous l'hypothèse $\mathrm{B}$ (cf. 3.26). Puis on transforme l'espérance du produit par la formule d'intégration par parties, et on constate leur égalité:

$$
\int_{0}^{T} E_{Q}\left(D_{t} F Z\left(H_{t}^{h}-H_{t}\right)\right) d t=\int_{0}^{T} E_{Q}\left(D_{t} F E_{Q}\left[Z\left(H_{t}^{h}-H_{t}\right) / \mathscr{G}_{T}^{Y}\right]\right) d t
$$

ce qui montre (4.58), sous réserve de vérifier la validité de (4.58) et (4.59), à savoir: l'appartenance de $E_{Q}\left[Z\left(H_{t}^{h}-H_{t}\right) / \mathscr{G}_{T}^{Y}\right]$ à $D_{q, 1}$ pour un $q>1$, qui donne un sens à l'intégrale à droite de (4.58) et permet d'appliquer (3.15). Cette appartenance est une conséquence du lemme A.1 montré en annexe dont on déduit de plus: 


$$
D_{s} E_{Q}\left[Z\left(H_{t}^{h}-H_{t}\right) / \mathscr{G}_{T}^{Y}\right]=E_{Q}\left[\left(H_{s}-\pi_{T}\left(H_{s}\right)\right) Z\left(H_{t}^{h}-H_{t}\right) / \mathscr{G}_{T}^{Y}\right]
$$

Pour démontrer (4.57) on utilise (4.58) auquel on applique la relation d'isométrie:

$$
\begin{aligned}
& E_{Q}\left[\delta\left(E_{Q}\left[Z\left(H^{h}-H\right) / \mathscr{G}_{T}^{Y}\right]\right)\right]^{2}=\int_{0}^{T} E_{Q}\left(E_{Q}\left[Z\left(H_{t}^{h}-H_{t}\right) / \mathscr{G}_{T}^{Y}\right]\right)^{2} d t \\
& +\iint E_{Q}\left(E_{Q}\left[\left(H_{s}-\pi_{T}\left(H_{s}\right)\right) Z\left(H_{t}^{h}-H_{t}\right) / \mathscr{G}_{T}^{Y}\right]\right. \\
& \left.\quad \times E_{Q}\left[\left(H_{t}-\pi_{T}\left(H_{t}\right)\right) Z\left(H_{s}^{h}-H_{s}\right) / \mathscr{G}_{T}^{Y}\right]\right) d s d t
\end{aligned}
$$

Remarquons que le deuxième terme, compte tenu de la symétrie en $s$ et $t$, est majoré par:

$$
\iint E_{\boldsymbol{Q}}\left(E_{\boldsymbol{Q}}\left[\left(H_{s}-\pi_{T}\left(H_{s}\right)\right) Z\left(H_{t}^{h}-H_{t}\right) / \mathscr{G}_{T}^{Y}\right]\right)^{2} d s d t
$$

On applique alors le lemme A.2 avec $F$ égal à $Z$, puis $Z H_{s}$ et $Z \pi_{T}\left(H_{s}\right)$ pour obtenir (4.57).

Pour traiter le second terme de (4.56), on étudie la $\mathscr{G}_{T}^{X}$-espérance conditionnelle de $\left(\delta\left(A^{h}-1\right)\left(H^{h}-H\right)\right)^{2}$ et l'on montre dans ce but le lemme suivant:

Lemme 4.8. Soit un processus $u$ appartenant à $D_{p, 1}(L), p>1$. Alors

$$
\begin{aligned}
& E\left[\delta_{t}(u) / \mathscr{G}_{T}^{X}\right]=0 \text { pour tout } t \text { et: } \\
& E\left[\left(\delta_{t}(u)-\delta_{S}(u)\right) / \mathscr{G}_{S}\right]=0 \text { pour } 0 \leq s \leq t \leq T
\end{aligned}
$$

En particulier, si le processus $\delta_{t}(u)$ est $\mathscr{F}_{t}$-mesurable, avec $\mathscr{F}_{t}=\mathscr{G}_{T}^{X} \vee \mathscr{G}_{t}$, alors c'est une $(\mathscr{F}, \boldsymbol{Q})$-martingale.

Démonstration. On a déjà montré (4.63) précédemment en (4.30). L'autre relation se montre de façon analogue en considérant une fonctionnelle $F \mathscr{G}_{s}$-mesurable et bornée, par exemple de la forme:

$$
F=f\left(X_{t_{i}}, B_{s_{j}} ; t_{i} \text { et } s_{\jmath} \leq s\right)
$$

avec $f$ de classe $C^{\infty}$ à dérivées bornées. On tire des règles de dérivation:

$$
D_{r} F=\partial_{j} f(\omega) \mathbf{1}_{\left[0, S_{j}\right]}(r)
$$

et de la formule d'intégration par parties:

$$
E\left[F\left(\delta_{t}(u)-\delta_{s}(u)\right)\right]=\int u_{r} D_{r} F d r=0
$$

ce qui montre (4.64). 
On est alors en mesure de montrer:

LEMME 4.9. $\quad E\left[\left(\delta\left(A^{h}-1\right)\left(H^{h}-H\right)\right)^{2} / \mathscr{G}_{T}^{X}\right]$

$$
\leq \int_{0}^{T}\left\|H_{t}^{h}-H_{t}\right\|^{2} E\left[\left(A_{t}^{h}-1\right)^{2} / \mathscr{G}_{T}^{X}\right] d t+E\left[\left(A^{*}\right)^{2} \mid \mathscr{G}_{T}^{X}\right]\left(\int_{0}^{T}\left\|H_{t}^{h}-H_{t}\right\|^{2} d t\right)^{2}
$$

où $A^{*}$ désigne le sup de $A_{s}^{h}$ sur $[0, T]$.

Démonstration. On tire de (4.29), pour tout $t$ :

$$
\delta_{t}\left(\left(A^{h}-1\right)\left(H^{h}-H\right)\right)=A_{t}^{h}-1-\delta_{t}\left(H^{h}-H\right)
$$

Sous l'hypothèse présente, $H^{h}-H$ appartient à tout $D_{q, k}(L)$. Par (3.14), il en est de même pour $\delta\left(H^{n}-H\right)$. Or $A^{n}-1$ appartient à $D_{p, 2}(L)$ (lemme 4.6) pour un $p>2$. Donc la somme, soit $\delta\left(\left(A^{n}-1\right)\left(H^{n}-H\right)\right)$, appartient à $D_{p, 2}(L)$ et le produit $\left(A^{h}-1\right)\left(H^{h}-H\right) \delta\left(\left(A^{h}-1\right)\left(H^{h}-H\right)\right)$ est un élément de $D_{r, 2}(L)$ avec $1 / r=1 / p+1 / q+1 / p$ grâce au lemme 3.1: c'est un élément intégrable au sens de Skorohod et on peut appliquer la proposition 3.5 à $\left(A^{h}-1\right)\left(H^{h}-H\right)$ :

$$
\begin{aligned}
& {\left[\delta\left(A^{h}-1\right)\left(H^{h}-H\right)\right]^{2}} \\
& =2 \int_{0}^{T}\left(A_{s}^{h}-1\right)\left(H_{s}^{h}-H_{s}\right) \delta_{s}\left(A^{h}-1\right)\left(H^{h}-H\right) d B_{s} \\
& \quad+\int_{0}^{T}\left(A_{s}^{h}-1\right)^{2}\left\|H_{s}^{h}-H_{s}\right\|^{2} d s \\
& \quad+2 \int_{0}^{T}\left(A_{s}^{h}-1\right)\left(H_{s}^{h}-H_{s}\right)\left(\int_{0}^{s} A_{r}^{h}\left(H_{s}^{h}-H_{s}\right)\left(H_{r}^{h}-H_{r}\right) \delta B_{r}\right) d s
\end{aligned}
$$

où l'on a remplacé $D_{s}\left(\left(A^{h}-1\right)\left(H^{h}-H\right)\right)$ par sa valeur (4.43) et là encore, on a omis sommations et indices des vecteurs $H$ et $B$. On applique alors le lemme 4.8 au premier terme de cette somme, dont l'intégrande appartient à $D_{r, 2}(L)$; il vient:

$$
\begin{aligned}
& E\left[\left(\delta\left(A^{h}-1\right)\left(H^{h}-H\right)\right)^{2} / \mathscr{G}_{T}^{X}\right]=\int_{0}^{T}\left\|H_{s}^{h}-H_{s}\right\|^{2} E\left[\left(A_{s}^{h}-1\right)^{2} / \mathscr{G}_{T}^{X}\right] d s \\
& \quad+2 \int_{0}^{T}\left(H_{s}^{h}-H_{s}\right) E\left[\left(A_{s}^{h}-1\right) \int_{0}^{s} A_{r}^{h}\left(H_{s}^{h}-H_{s}\right)\left(H_{r}^{h}-H_{r}\right) \delta B_{r} / \mathscr{G}_{T}^{X}\right] d s
\end{aligned}
$$

où l'on a utilisé que $H$ est $\mathscr{G}_{T}^{X}$-mesurable. Sous l'espérance conditionnelle ci-dessus, on peut faire "entrer" $A_{s}^{h}-1$ dans l'intégrale de Skorohod à l'aide de la relation (3.20):

$$
\begin{aligned}
& \left(A_{s}^{h}-1\right) \int_{0}^{s} A_{r}^{h}\left(H_{s}^{h}-H_{s}\right)\left(H_{r}^{h}-H_{r}\right) \delta B_{r} \\
& \quad=\int_{0}^{s} \sigma_{r}^{s} \delta B_{r}+\int_{0}^{s} A_{r}^{h} A_{s}^{h}\left(H_{r}^{h}-H_{r}\right)\left\|H_{r}^{h}-H_{r}\right\|^{2} d r
\end{aligned}
$$


où l'on a encore utilisé (4.43), et où $\sigma^{s}=\left(A_{s}^{h}-1\right) A^{h}\left(H^{h}-H\right)\left(H_{s}^{h}-H_{s}\right)$ est un élément de $D_{r, 1}(L)$ avec $r>1$; le lemme 4.8 permet d'annuler la $\mathscr{G}_{T^{-}}^{X}$ espérance conditionnelle de $\delta\left(\sigma^{s} \mathbf{1}_{[0, s]}\right)$. Par ailleurs, le processus $A^{h}$ est positif et le produit $A_{s}^{h} A_{r}^{h}$ est uniformément majoré en $\left(A^{*}\right)^{2}$. On déduit donc de (4.70)-(4.71):

$$
\begin{gathered}
E\left[\left(\delta\left(A^{h}-1\right)\left(H^{h}-H\right)\right)^{2} / \mathscr{G}_{T}^{X}\right] \leq \int_{0}^{T}\left\|H_{s}^{h}-H_{s}\right\|^{2} E\left[\left(A_{s}^{h}-1\right)^{2} / \mathscr{G}_{T}^{X}\right] d s \\
\quad+2 \int_{0}^{T}\left\|H_{s}^{h}-H_{s}\right\|^{2} \int_{0}^{s}\left\|H_{r}^{h}-H_{r}\right\|^{2} E\left[\left(A^{*}\right)^{2} / \mathscr{G}_{T}^{X}\right] d s d r,
\end{gathered}
$$

ce qui achève la démonstration du lemme.

Lemme 4.10. Pour tout $t$ de $[0, T]$,

$$
E\left[\left(A_{t}^{h}-1\right)^{2} / \mathscr{G}_{T}^{X}\right] \leq \varepsilon(h)(1+\varepsilon(h)) E\left[\left(A^{*}\right)^{2} / \mathscr{G}_{T}^{X}\right]
$$

où $\varepsilon(h)$ désigne la variable $\int_{0}^{T}\left\|H_{t}^{h}-H_{t}\right\|^{2} d t$.

Démonstration. Le processus $A^{h}-1$ n'est autre que $\delta\left(A^{h}\left(H^{h}-H\right)\right)$. On peut donc appliquer la formule de Itô élargie à son carré comme on vient de le faire pour $\left(A^{h}-1\right)\left(H^{h}-H\right)$ :

$$
\begin{aligned}
\left(A_{t}^{h}-1\right)^{2}= & 2 \int_{0}^{T}\left(A_{s}^{h}-1\right) A_{s}^{h}\left(H_{s}^{h}-H_{s}\right) \delta B_{s}+\int_{0}^{\iota}\left(A_{s}^{h}\right)^{2}\left\|H_{s}^{h}-H_{s}\right\|^{2} d s \\
& +2 \int_{0}^{t} A_{s}^{h}\left(H_{s}^{h}-H_{s}\right)\left(\int_{0}^{s} A_{r}^{h}\left(H_{s}^{h}-H_{s}\right)\left(H_{r}^{h}-H_{r}\right) \delta B_{r}\right) d s
\end{aligned}
$$

Des calculs identiques à (4.69)-(4.72) conduisent à la majoration:

$$
\begin{aligned}
E\left[\left(A_{t}^{h}-1\right)^{2} / \mathscr{G}_{T}^{X}\right] & \leq \int_{0}^{t}\left\|H_{s}^{h}-H_{s}\right\|^{2} d s E\left[\left(A^{*}\right)^{2} / \mathscr{G}_{T}^{X}\right] \\
& +2 \int_{0}^{t}\left\|H_{s}^{h}-H_{s}\right\|^{2} \int_{0}^{s}\left\|H_{r}^{h}-H_{r}\right\|^{2} d r d s E\left[\left(A^{*}\right)^{2} / \mathscr{G}_{T}^{X}\right]
\end{aligned}
$$

Cette dernière majoration implique (4.73).

LEMME 4.11. $\quad\left\|\delta\left(A_{t}^{h}-1\right)\left(H^{h}-H\right)\right\|_{2}=0(h)$.

Démonstration. On a à calculer l'espérance:

$$
E\left(\delta\left(A^{h}-1\right)\left(H^{h}-H\right)\right)^{2}=E\left(E\left[\left(\delta\left(A^{h}-1\right)\left(H^{h}-H\right)\right)^{2} / \mathscr{G}_{T}^{X}\right]\right)
$$

Les lemmes 4.9 et 4.50 donnent la majoration:

$$
E\left[\left(\delta\left(A^{h}-1\right)\left(H^{h}-H\right)\right)^{2} / \mathscr{G}_{T}^{X}\right] \leq(\varepsilon(h))^{2}(2+\varepsilon(h)) E\left[\left(A^{*}\right)^{2} / \mathscr{G}_{T}^{X}\right]
$$


Il s'agit donc d'étudier $E\left[\left(A^{*}\right)^{2} / \mathscr{G}_{T}^{X}\right]$. Or, si $\mathscr{F}$ est la filtration définie au lemme $4.8\left(\mathscr{F}_{t}=\mathscr{G}_{T}^{X} \vee \mathscr{G}_{t}\right)$, on peut montrer que $A^{h}$ est $\mathscr{F}$-adapté. En effet, $A^{h}$ est le produit $L^{h} L$, avec $L$ processus $\mathscr{G}$-adapté (2.6); de plus, si l'on revient à la définition (2.15) de $L^{h}$, on obtient pour $t$ dans $I_{i}$ :

$$
L_{t}^{h}=L_{(i-1) h}^{h} \exp \int_{(i-1) h}^{t} H_{i h} \delta B_{s} \exp \int_{(i-1) h}^{t}\left(H_{i h} H_{s}-1 / 2\left\|H_{i n}\right\|^{2}\right) d s
$$

Le facteur $L_{(i-1) h}^{h}$ est $\mathscr{G}_{(i-1) h}$ (donc $\mathscr{G}_{t}$, donc $\mathscr{F}_{t}$ )-mesurable; le troisième facteur de $L^{h}$ ne dépend que de $H$, donc est $\mathscr{G}_{T}^{X}$-mesurable; quant au second, on le réécrit à l'aide de la relation (3.20), sachant que $D H$ est nul:

$$
\exp \int_{(i-1) h}^{t} H_{i h} \delta B_{s}=\exp H_{i h}\left(B_{t}-B_{(i-1) h}\right)
$$

qui est $F_{t}$-mesurable et $A^{h}$ est bien $\mathscr{F}$-adapté. Puisque $A^{h}$ est également un processus de $D_{p, 1}(L)$, le lemme 4.8 montre que $A^{h}$ est une $(\mathscr{F}, \boldsymbol{Q})$ martingale, de tribu initiale $\mathscr{G}_{T}^{X}$. On lui applique l'inégalité de Doob:

$$
E\left[\left(A^{*}\right)^{2} / \mathscr{G}_{T}^{X}\right] \leq 4 E\left[\left(A_{T}^{h}\right)^{2} / \mathscr{G}_{T}^{X}\right]
$$

Grâce au lemme 4.5, la variable majorante appartient à $L^{p / 2}$ et on tire de (4.77), (4.80) et d'une inégalité de Hölder:

$$
E\left(\delta\left(A^{h}-1\right)\left(H^{h}-H\right)\right)^{2} \leq 4 \|\left(\varepsilon ( h ) ^ { 2 } ( 2 + \varepsilon ( h ) ) \| _ { q } \| E \left[\left(A_{T}^{h}\right)^{2} / \mathscr{G}_{T}^{X} \|_{p / 2}\right.\right.
$$

avec $1 / q+2 / p=1$. Le premier facteur de cette majoration est un $0\left(h^{2}\right)$ grâce au lemme 4.2; le second est uniformément majoré en $h$ par $M$ (lemme 4.5), ce qui achève la démonstration du lemme.

Ainsi (4.2) est-il démontré en rapprochant le lemme 4.11 des relations (4.56), (4.57) et (4.4).

\section{§ V. Conclusion}

Le lemme 4.11 et l'estimation 4.57 utilisent de façon cruciale l'indépendance de la fonction du signal et du bruit. C'est pourquoi il est difficile d'espérer que l'approximation en $0(h)$ dans le cas de l'indépendance puisse se généraliser à des fonctions du signal dépendant de l'obervation. Ceci dit, le recours au calcul stochastique permet, même dans le cas où la fonction du signal est indépendante du bruit, une formalisation agréable et des calculs relativement simples, le plus souvent grâce à la formule d'intégration par parties. 


\section{Annexe}

LEMmE A.1. Soit $Z$ une fonctionnelle $\mathscr{G}_{T}^{X}$-mesurable admettant des moments de tout ordre. Alors, sous les hypothèses (2.4) B, (2.5) et (3.33), $E_{Q}\left[Z / \mathscr{G}_{T}^{Y}\right]$, soit $\pi_{T}(Z)$, appartient à $D_{q, 1}$, pour tout $q>1$ et

$$
D\left(\pi_{T}(Z)(h)\right)=\int_{0}^{T} \pi_{T}\left[\left(H_{s}-\pi_{T}\left(H_{s}\right)\right) Z\right] h_{s} d s, \quad \forall h \in L .
$$

Démonstration. Pour montrer l'appartenance de $\pi_{T}(Z)$ à $D_{q, 1}$ on peut vérifier le critère de Sugita (cf. (3.3) et sq) c'est-à-dire l'existence de la limite en probabilité du quotient:

$$
\left[\pi_{T}(Z)\left(B+\varepsilon \int_{0} h_{s} d s\right)-\pi_{T}(Z)(B)\right] / \varepsilon
$$

Sous les hypothèses du lemme, translater les trajectories de $B$ ou de $Y$ est équivalent grâce à la définition (1.1). De plus, les probabilités $\boldsymbol{P}$ et $\boldsymbol{Q}$ étant équivalentes, on peut étudier la limite en probabilité de (A.2) aussi bien sous $\boldsymbol{P}$ que sous $\boldsymbol{Q}$. On réécrit $\pi_{T}(Z)$ sur $(\Omega, P)$ comme quotient, en mettant en évidence la dépendance en $Y$ et en utilisant $\boldsymbol{P}=$ $\boldsymbol{P}^{X} \otimes \boldsymbol{P}^{Y}:$

$$
\begin{aligned}
& \pi_{T}(Z)=\sigma_{T}(Z) / \sigma_{T}(1) \\
& \sigma_{T}(Z)=E^{X}\left[Z \exp \left(H_{T} Y_{T}-\int_{0}^{T} Y_{s} d H_{s}+1 / 2 \int_{0}^{T}\left\|H_{s}\right\|^{2} d s\right)\right]
\end{aligned}
$$

L'expression (A.2) devient:

$$
\begin{aligned}
& {\left[\sigma_{T}\left(Z \exp \varepsilon \int_{0}^{T} H_{s} h_{s} d s\right) / \sigma_{T}\left(\exp \varepsilon \int_{0}^{T} H_{s} h_{s} d s\right)-\pi_{T}(Z)\right] / \varepsilon } \\
= & \frac{\sigma_{T}\left(Z\left(\exp \varepsilon \int_{0}^{T} H_{s} h_{s} d s-1\right) / \varepsilon\right)}{\sigma_{T}\left(\exp \varepsilon \int_{0}^{T} H_{s} h_{s} d s\right)}+\frac{\sigma_{T}(Z) \sigma_{T}\left(\exp \varepsilon \int_{0}^{T} H_{s} h_{s} d s-1\right) / \varepsilon}{\sigma_{T}(1) \sigma_{T}\left(\exp \varepsilon \int_{0}^{T} H_{s} h_{s} d s\right)}
\end{aligned}
$$

Numérateur et dénominateur de ces deux quotients convergent presque sûrement, grâce au théorème de convergence monotone et donc (A.4) admet a fortiori une limite en probabilité, qui est exactement le membre à droite de (A.1).

Pour conclure de lemme, il suffit de remarquer que, d'une part cette limite permet de définir:

$$
D_{s}\left(\pi_{T}(Z)\right)=\pi_{T}\left[\left(H_{s}-\pi_{H}\left(H_{s}\right)\right) Z\right],
$$


élément de $L$ comme $\left(H-\pi_{T}(H)\right) Z$ dont il est une version de l'espérance conditionnelle; et d'autre part que la norme dans $L$ de (A.5) admet des moments de tout ordre comme $Z$ et $H$.

Lemme A.2. Soit $F$ une fonctionnelle de $L$, soit $\mathscr{G}_{T}^{X}$-mesurable et de L-norme dans tout $L^{p}$, soit appartenant à $D_{q, 1}(L)$ pour tout $q>1$, à un ccefficient mesurable et borné près. Alors

$$
\int_{0}^{T} \int_{0}^{T}\left\|E_{Q}\left[F_{s}\left(H_{t}^{h}-H_{t}\right) / \mathscr{G}_{T}^{Y}\right]\right\|_{2}^{2} d t d s=0\left(h^{2}\right) .
$$

\section{Démonstration.}

1. On met d'abord en évidence l'existence d'un processus $\varphi$ tel que, si $t \in I_{i}$ :

$$
\begin{aligned}
& E_{Q}\left[F_{s}\left(H_{t}^{h}-H_{t}\right) / \mathscr{G}_{T}^{Y}\right]=E_{Q}\left[\int_{t}^{i h} \varphi_{u, s} d u / \mathscr{G}_{T}^{Y}\right] \\
& \varphi_{u, s}=F_{s} b_{u}+\sigma_{u}\left(D_{u}^{X} F_{s}+F_{s} \int_{0}^{T} D_{u}^{X} H_{v} d B_{v}\right)
\end{aligned}
$$

Soit en effet une fonctionnelle $G, \mathscr{G}_{T}^{Y}$-mesurable et bornée; on calcule l'espérance du produit de $G$ avec (A.7), en utilisant l'expression (2.5) de $H$ si $t \in I_{i}$ :

$$
E_{Q}\left[G F\left(H_{t}^{h}-H_{t}\right)\right]=E_{Q}\left[G \int_{t}^{i n} F b_{u} d u\right]+E_{Q}\left[G F \int_{t}^{i n} \sigma_{u} d V_{u}\right]
$$

Le premier terme à droite de (A.8) donne le premier terme de $\varphi$. On calcule le second en utilisant la probabilité $\boldsymbol{P}=L_{T} \boldsymbol{Q}$ :

$$
E_{\mathbf{Q}}\left[G F \int_{t}^{i n} \sigma_{u} d V_{u}\right]=E_{\boldsymbol{P}}\left[L_{T}^{-1} G F \int_{t}^{i n} \sigma_{u} d V_{u}\right]
$$

Le lemme 3.7, les hypothèses sur $F$ et $\sigma$, l'indépendance de $X$ et $Y$ sous $\boldsymbol{P}$ permettent d'appliquer la formule d'intégration par parties relative aux composantes de $V$ :

$$
\begin{aligned}
& E_{P}\left[L_{T}^{-1} G F \int_{t}^{i n} \sigma_{u} d V_{u}\right] \\
& \quad=E_{P}\left[L_{T}^{-1} \int_{t}^{i n} G \sigma_{u}\left(D_{u}^{X} F+F \int_{0}^{T} D_{u}^{X} H_{v}\left(d Y_{v}-H_{v} d v\right)\right) d u\right] \\
& \quad=E_{Q}\left[G \int_{t}^{i n} \sigma_{u}\left(D_{u}^{X} F+F \int_{u}^{T} D_{u}^{X} H_{v} d B_{v}\right) d u\right]
\end{aligned}
$$

ce qui donne les deuxième et troisième termes de $\varphi$ et montre A.7. 
2. On montre ensuite que $\varphi$ est de carré intégrable sur $[0, T]^{2} \times \Omega$; c'est le cas des deux premiers termes: $\sigma$ et $b$ sont bornés, et par hypothèse, les normes de Hilbert-Schmidt de $F$ et $D^{x} F$ sont dans tout $L^{p}$. L'étude du troisième terme conduit à distinguer les deux cas du lemme. Lorsque $F$ est $\mathscr{G}_{T}^{X}$-mesurable, l'indépendance de $X$ et $B$ permet de faire "entrer" $F_{\sigma_{u}}$ sous l'intégrale stochastique:

$$
\sigma_{u} F \int_{u}^{T} D_{u}^{X} H_{v} d B_{v}=\int_{u}^{T} \sigma_{u} F D_{u}^{X} H_{v} d B_{v}
$$

dont l'espérance du carré est majorée.

$$
E_{Q}\left[\sigma_{u}^{2} F_{s}^{2}\left(\int_{u}^{T} D_{u}^{X} H_{v} d B_{v}\right)^{2}\right] \leq\|\sigma\|_{\infty}^{2} E_{Q} \int_{u}^{T} F_{s}^{2}\left\|D_{u}^{X} H_{v}\right\|^{2} d v
$$

Cette majoration admet comme intégrale sur $[0, T]^{2}$ l'espérance de $\|F\|_{L}^{2}\left\|D^{x} H\right\|_{\text {I.S. }}^{2}$, qui est finie puisque chacun des facteurs est dans tout $L^{p}$.

Lorsque $F$ est une fonctionnelle de $D_{q, 1}(L)$, on peut appliquer (3.20):

$$
\sigma_{u} F \int_{u}^{T} D_{u}^{X} H_{v} d B_{v}=\int_{u}^{T} \sigma_{u} F D_{u}^{X} H_{v} \delta B_{v}+\int_{u}^{T} \sigma_{u} D_{v} F D_{u}^{X} H_{v} d v
$$

Le coefficient $\sigma$ borné ne joue pas de rôle; on applique la relation d'isométrie au premier terme:

$$
\begin{aligned}
& E_{Q}\left(\int_{u}^{T} F_{s} D_{u}^{X} H_{v} \delta B_{v}\right)^{2} \\
& \quad=E_{\boldsymbol{Q}} \int_{u}^{T} F_{s}^{2}\left\|D_{u}^{X} H_{v}\right\|^{2} d v+E_{\boldsymbol{Q}} \int_{u}^{T} \int_{u}^{T} D_{t} F_{s} D_{u}^{X} H_{v} D_{v} F_{s} D_{u}^{X} H_{t} d t d v
\end{aligned}
$$

La symétrie en $t$ et $v$ de cette deuxième intégrale montre qu'elle est exactement l'espérance du carré du deuxième terme de (A.13) dont on peut majorer l'intégrale en $u$, après permutation:

$$
\begin{aligned}
& \left|\int_{0}^{T} \int_{u}^{T} \int_{u}^{T} D_{v} F_{s} D_{u}^{X} H_{v} D_{t} F_{s} D_{u}^{X} H_{t} d t d v d u\right| \\
& \quad=\left|\iint D_{v} F_{s} D_{t} F_{s}\left(\int D_{u}^{X} H_{v} D_{u}^{X} H_{t} d u\right) d t d v\right| \\
& \quad \leq \iint\left|D_{v} F_{s}\left\|D_{t} F_{s} \mid\right\| D^{X} H_{v}\|\| D^{X} H_{t}\left\|_{L} d t d v \leq\right\| D F_{s}\left\|_{2}^{2}\right\| D^{X} H \|_{\mathrm{H} . \mathrm{S} .}^{2}\right.
\end{aligned}
$$

qui est intégrable sur $[0, T] \times \Omega$ comme produit de facteurs appartenant à tout $L^{p}$. Quant au premier terme de (A.14), son intégrale sur $[0, T]^{2}$ est l'espérance de $\|F\|_{L}^{2}\left\|D^{x} H\right\|_{\text {H.s. }}^{2}$ qui est également finie. 
3. Dans ces deux cas, utilisant alors (A.7) on obtient:

$$
\iint E_{Q}\left\|E_{Q}\left[F_{s}\left(H_{t}^{h}-H_{t}\right) / \mathscr{G}_{T}^{X}\right]\right\|^{2} d s d t \leq \iint E_{\boldsymbol{Q}}\left[\int_{t}^{i n} \varphi_{u, s} d u\right]^{2} d s d t
$$

Considérant d'abord l'intégrale en $t$, fractionnée sur les $I_{i}$ :

$$
\int_{I_{i}} E_{Q}\left[\int_{t}^{i h} \varphi_{u, s} d u\right]^{2} d t \leq \int_{I_{i}}(i h-t) E_{Q}\left[\int_{t}^{i h} \varphi_{u, s}^{2} d u\right] d t \leq h^{2} / 2 E_{Q} \int_{I_{i}} \varphi_{u, s}^{2} d u
$$

La somme en $i$ de ces termes, puis l'intégrale en $s$, est l'espérance de $\varphi^{2}$ sur $[0, T]^{2} \times \Omega$ au coefficient $h^{2} / 2$ près, ce qui montre (A.6) pour ces deux cas.

Remarquons enfin que si $F_{s}$ est multiplié par un coefficient $Z$ borné, on a:

$$
\left\|E_{Q}\left[Z F_{s}\left(H_{t}^{h}-H_{t}\right) / \mathscr{G}_{T}^{X}\right]\right\| \leq M\left\|E_{Q}\left[F_{s}\left(H_{t}^{h}-H_{t}\right) / \mathscr{G}_{T}^{X}\right]\right\|
$$

ce qui achève la démonstration du lemme.

Remerciements. Que soient ici remerciés les professeurs Etienne Pardoux, Süleyman Ustünel, Dominique Michel, Dominique Lépingle et David Nualart pour l'aide qu'ils m'ont apportée aux différentes étapes de ce travail. Je remercie également le rapporteur anonyme de ses commentaires et de la lecture attentive qu'il a faite de ce papier.

\section{REFERENCES}

[1] C. Dellacherie - P. Meyer, "Probabilités et Potentiels", T1 \& 2, Hermann, Paris, 1980.

[2] G. B. Di Masi - M. Pratelli - W. J. Runggaldier, "An approximation for the nonlinear filtering problem with error bound", Stochastics, 15 (1985), 247-271.

[ 3 ] N. Ikeda - S. Watanabe, "Stochastic Differential Equations and Diffusion Process", North Holland, Amsterdam, 1981.

[4] —-, "Malliavin calculus of Wiener functionals and its applications", Warwick Symposium on "Stochastic Differential Equations and Applications", From local times to global geometry, control and physics, edited by K. D. Elworthy, Pitman Research Notes in Math. Series 150, Longman Scientific and Technical, New York, 1986.

[5] H. Korezlioglu - G. Mazziotto, "Modélisation et filtrage de systèmes stochastiques non linéaires" Note technique CNET, 1983.

[6] A. Millet-D. Nualart-M. Sanz, "Integration by parts and time reversal for diffusion processes", Preprint, 1987.

[ 7 ] D. Nualart, "Non causal stochastic integral and calculus", Workshop on Stochastic Analysis, Silivri (Istanbul), July 1986, L.N. in Maths $n^{\circ} 1316$, Springer-Verlag, New York, 1988.

[ 8 ] D. Nualart-M. Zakai, "Generalized stochastic integrals and the Malliavin calculus", Probab. Theory, 73 (1986), 255-280. 
[ 9 ] E. Pardoux - P. Protter, "Two sided-stochastic Integrals", Probab. Theory, 76-1 (1987), 15-50.

[10] J. Picard, "An estimate of the error in time discretization of nonlinear filtering problems", $7^{\text {th }}$ MT NS Symposium, Stockholm, 1985.

[11] M. Pontier, "Approximation d'un filtre avec observation sur une variété compacte", Stochastics, 24 (1988), 285-304.

[12] H. Sugita, "On a characterization of Sobolev Spaces over an abstract Wiener space", J. Math. Kyoto Univ., 25 (1985), 717-725.

[13] A. S. Ustunel, "The Itô formula for anticipative processes with non monotonous time scale via the Malliavin calculus", Theory of Probability and related fields, 79 (1988), 249-269.

[13b] _ - "Representation of the distributions on Wiener Space and Stochastic Calculus of Variations", J. of Funct. Anal., 70 (1987), 126-139.

[14] S. Watanabe, "Stochastic Differential Equation and Malliavin calculus", Tata Institute of Fundamental Research, Bombay, 1984.

[15] M. Zakai, "The Malliavin calculus", Acta Appl. Math., 3 (1985), 175-207.

[16] S. Taniguchi, "Malliavin's Stochastic calculus of Variations for Manifold-Valued Wiener Functionals and its Applications", Z. Wahrsch., 65 (1983), 269-290.

[17] M. H. A. Davis, "The Wiener space derivative for functionals of diffusions on manifolds", Nonlinearity, 1, United Kingdom, 1988, 241-251.

Département "Mathématiques \& Informatique"

Université d'Orléans

B. P. 6759

45067 , Orléans Cedex 2

France 